\title{
Climate change and mosquito-borne diseases in China: a review
}

Li Bai ${ }^{1}$, Lindsay Carol Morton ${ }^{2}$ and Qiyong Liu ${ }^{1,3^{*}}$

\begin{abstract}
China has experienced noticeable changes in climate over the past 100 years and the potential impact climate change has on transmission of mosquito-borne infectious diseases poses a risk to Chinese populations. The aims of this paper are to summarize what is known about the impact of climate change on the incidence and prevalence of malaria, dengue fever and Japanese encephalitis in China and to provide important information and direction for adaptation policy making. Fifty-five papers met the inclusion criteria for this study. Examination of these studies indicates that variability in temperature, precipitation, wind, and extreme weather events is linked to transmission of mosquito-borne diseases in some regions of China. However, study findings are inconsistent across geographical locations and this requires strengthening current evidence for timely development of adaptive options. After synthesis of available information we make several key adaptation recommendations including: improving current surveillance and monitoring systems; concentrating adaptation strategies and policies on vulnerable communities; strengthening adaptive capacity of public health systems; developing multidisciplinary approaches sustained by an new mechanism of inter-sectional coordination; and increasing awareness and mobilization of the general public.
\end{abstract}

Keywords: Climate change, Malaria, Dengue fever, Japanese encephalitis, Adaptation, China

\section{Introduction}

The Intergovernmental Panel on Climate Change (IPCC) has reported the existence of abundant evidence of climate change on a global scale [1]. According to the IPCC's fourth assessment in 2007, global average surface temperature will increase by $1.1-6.4^{\circ} \mathrm{C}$ by $2100,2-9$ times more than globally averaged warming during last century [1]. Furthermore, the frequency and extent of extreme weather events; such as heat waves, bushfires, floods, and cyclones, can be highly impacted by the changing climate. Anthropogenic climate change has also been identified as an important risk factor for population health [2], including transmission of infectious diseases, and most importantly suspected impacts distribution and occurrence of vector borne diseases [1]. Despite the ongoing debate over the influence climate

\footnotetext{
* Correspondence: liuqiyong@icdc.cn

${ }^{1}$ State Key Laboratory for Infectious Diseases Prevention and Control, National Institute for Communicable Disease Control and Prevention, Chinese Center for Disease Control and Prevention, Beijing 102206, People's Republic of China

${ }^{3}$ Shandong University Climate Change and Health Center, Jinan 250012Shandong, People's Republic China

Full list of author information is available at the end of the article
}

factors have on mosquito-borne disease occurrence, it is widely assumed that distribution and occurrence of these diseases, such as malaria, are determined by climate and that global warming trends will lead to higher incidence and wider geographic range [3-10]. In contrast, some studies hold that the current evidence is insufficient to clearly attribute local resurgences or such geographic spread to regional changes in climate [11-14]. More research is needed to better understand the relationship between climate change and transmission of mosquito-borne diseases, and to further promote adaptive policies formulation to reduce unexpected climate-related risk at a global, regional or local level.

Mosquito-borne diseases in China remain a serious public health problem. For example, 46,988 malaria cases and 18 deaths were reported in 1,097 counties in 2007 [15]. In 2002, the most serious outbreak of dengue fever occurred in Taiwan with 5,285 diagnosed cases [16]. In 2006, an outbreak of Japanese encephalitis occurred in Shanxi Province causing 19 deaths [17]. As the largest developing country, China has experienced considerable changes in climate over during the last decade with more rapid changes in the past 50 years [18]. The 
annual average temperature has risen by $0.5-0.8^{\circ} \mathrm{C}$, which is slightly higher than the global average level. These variation and fluctuation in weather patterns and extreme climatological phenomena (e.g. droughts, storms, floods etc.) may have a detrimental effect on frequency and distribution of mosquito-borne diseases.

In recent years, the impact of climate change on the transmission of mosquito-borne diseases has been studied in China. However, the quantitative relationship between meteorological variables and the spatial and temporal distributions of these infectious diseases is still not clear. Study findings are inconsistent, which may be due to methodological limitations, unavailability of relevant data and many uncertainties about the range and magnitude of influences of climate change. Moreover, there remains no adaptive mechanism to reduce adverse consequences of mosquito-borne diseases under the changing climate in China. It is urgent to improve our understanding of current evidence, knowledge gaps and development of adaptation options. Our aims were to summarize previous research exploring climate changerelated impacts on mosquito-borne diseases in China by reviewing the published studies examining the relationship between climate variability and the transmission of malaria, dengue fever and Japanese encephalitis, and to give some suggestions for the development of adaptation strategies and measures to lessen the adverse impacts on mosquito-borne diseases in China.

\section{Methods}

\section{Search strategy}

The PubMed electronic database and China Hospital Knowledge Database (CHKD) were used in December
2011 to retrieve original studies published in English and Chinese, respectively. Searches of the "Google" search engine and "Google Scholar" were also conducted. Combinations of the key terms "malaria", "dengue", "dengue fever", "dengue hemorrhagic fever", "Japanese encephalitis", "climate", "weather", "climate change", "climate variability", "climatic factors", "temperature", "rainfall", and "humidity" were used to maximize search yield. Titles, abstracts and keywords were first screened for relevance and full texts were obtained to evaluate for inclusion criteria. Reference lists of each included article were then evaluated if missed in the in the initial electronic database search. Figure 1 illustrates the systematic search and inclusion/exclusion process.

\section{Inclusion criteria}

Studies were included on the basis of the following criteria:

1. Articles must evaluate the effects of climatic factors on the distribution and transmission of malaria, dengue fever or Japanese encephalitis. Meteorological variables (e.g. temperature, rainfall etc.) or ecological proxy indicators (e.g. Normalized Difference Vegetation Index, South Oscillation Index) had to be included. Disease variables (e.g. incidence, cases) or entomologic variables (e.g. Breteau Index, House Index etc.) had to be included.

2. The papers had to use an epidemiological study design (e.g. time series analysis, spatial and/or temporal analysis and descriptive study) to identify the association between climatic variables and incidence of mosquito-borne diseases and/or vector

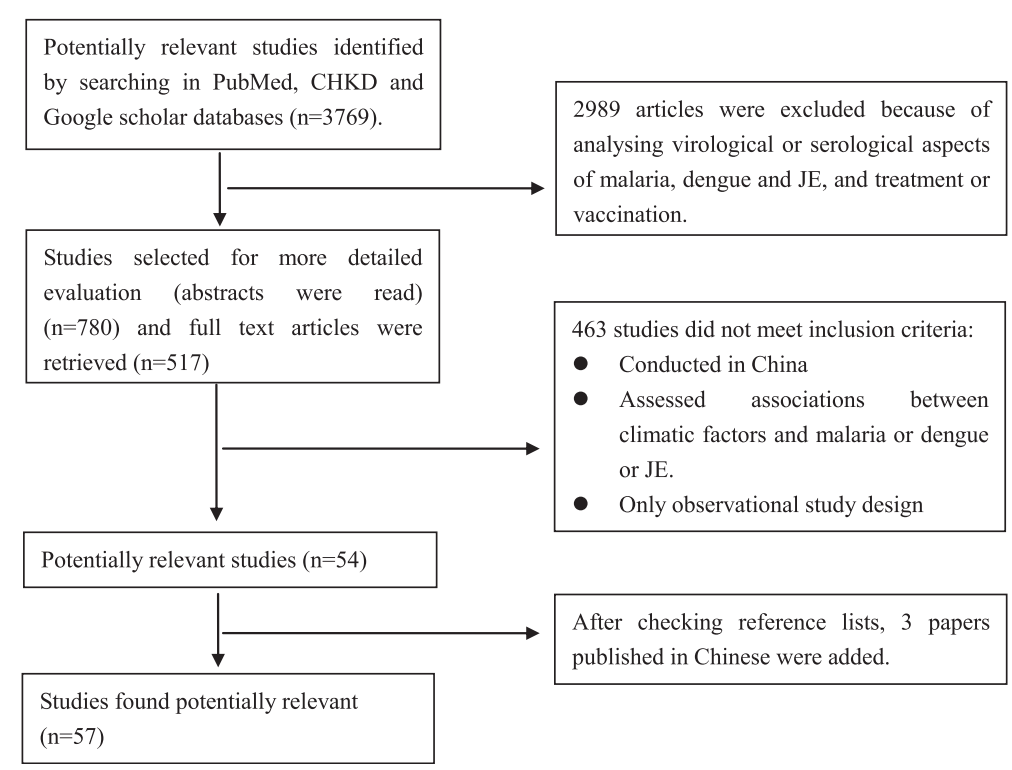

Figure 1 Flow chart of literature search strategy. 
factors (e.g. mosquito density, distribution, infectious life span).

3. Only studies published before December 2012 and conducted in China (including Chinese Autonomous Regions, Hong Kong, Macau and Taiwan) were examined.

\section{Results and discussion Literature search}

To avoid language bias, studies published in English and Chinese were considered for inclusion (Table 1). The initial search generated 3769 articles from PubMed, CHKD and Google Scholar databases. Review of the titles, abstracts and keywords excluded 2989 articles, leaving 780 studies identified as potential epidemiological papers. Then, 517 full-text articles were identified based on the abstracts and evaluated for inclusion. Of these, fifty-four articles met the inclusion criteria and 3 papers were included after cross-referencing. The methodologies and major findings of the final 57 studies are summarized in Tables 2, 3 and 4 .

Study sites mainly included Yunnan Province $(\mathrm{n}=9)$, Hainan Province $(n=8)$, Anhui Province $(n=7)$, Taiwan $(\mathrm{n}=7)$, Guizhou Province $(\mathrm{n}=5)$, Shandong Province $(\mathrm{n}=3)$, Guangdong Province $(\mathrm{n}=3)$, Henan Province $(n=2)$, Hubei Province $(n=2)$, Jiangsu Province $(n=2)$, and Liaoning Province $(\mathrm{n}=2)$. Other locations included Jiangxi Province, Hebei Province, Shanxi Province, Shaanxi Province, Sichuan Province, Gansu Province, Zhejiang Province, Fujian Province, Beijing Municipality, Chongqing Municipality, Tianjin Municipality, Inner Mongolian Autonomous Region, and Tibetan Autonomous Region. However, there was no study conducted in some regions where mosquito-borne diseases are endemic, such as Guangxi and Hunan Province.

All included studies examined the relationship between climatic variables and mosquito-borne diseases. Of these, only 6 studies evaluated of the impacts of both meteorological factors and other relevant determinants, such as urbanization, agriculture and vaccination $[51,54,64,71,73]$. Several studies used ecological proxy indicators including Normalized Difference Vegetation Index (NDVI) and South Oscillation Index (SOI) as risk variables $[28,29,35,37,39,43]$.

Table 1 Numbers of selected studies published in English and Chinese

\begin{tabular}{lccc}
\hline & $\begin{array}{c}\text { Studies in English } \\
\text { Published between } \\
\text { 1998-2011 }\end{array}$ & $\begin{array}{c}\text { Studies in Chinese } \\
\text { Published between } \\
\text { 1995-2011 }\end{array}$ & Total \\
\hline Malaria & 13 & 15 & $\mathbf{2 8}$ \\
\hline Dengue fever & 9 & 6 & $\mathbf{1 5}$ \\
\hline Japanese encephalitis & 4 & 10 & $\mathbf{1 4}$ \\
\hline Total & $\mathbf{2 6}$ & $\mathbf{3 1}$ & $\mathbf{5 7}$ \\
\hline
\end{tabular}

Two studies explored the influence of typhoons on outbreaks of dengue fever $[48,52]$. The main outcome indicators evaluated in this review were case count and incidence rate. Several studies collected data on entomologic factors $[38,47,48,60]$. Only 7 studies evaluated both disease and mosquito data together $[21,48,49,54,55,59,61]$.

A variety of methods were used to determine the effects of climatic variables on diseases and mosquitoes. Among them, 9 employed spatial study designs [23,31, $35,39,43,47,48,54,56], 6$ time-series analyses [20,22,27, $30,55,62]$, and 4 spatial-temporal methods [19,24-26]. Simple comparisons between climate, disease and/or vector data were applied in 10 descriptive studies [34,45, $53,57,58,60,61,68,73,75]$. Correlations and multiple regressions (e.g logistic and Poisson regression) were widely conducted among selected articles to examine associations between weather parameters and mosquito-borne diseases. Time-series models including autoregressive integrated moving average (ARIMA) model and seasonal ARIMA model were mainly used in recently published studies. Risk maps of mosquito-borne diseases and vector distribution in different regions were also present in some articles using spatial analysis with Geographic Information System (GIS) $[23,47,56]$. Other statistical methods used included study designs utilizing Principle Component Analysis (PCA), Back propagation artificial neural network and CLIMEX model etc.

\section{Associations between climatic variables and mosquito- borne diseases Malaria}

Despite considerable reductions in the overall burden of malaria in the 20th century, this ancient disease still represents a significant public health problem in China, especially in the southern and central regions. In 2010, 7,855 diagnosed malaria cases and 34,082 suspected cases with 19 deaths were reported in 1191 counties of 239 Provinces/Municipalities/Autonomous Regions in China. The annual incidence was $0.66 / 10,000$ population [76]. Only sixteen percent of malaria cases were caused by Plasmodium falciparum [76] mostly occurring in Yunnan Province, which is located in southern China. Yunnan remains a hypo-endemic region with persistent cases of both $P$. falciparum and $P$. vivax malaria.

To identify risk factors related to climate change and its role in malaria transmission, a series of studies were conducted in mainland China to investigate the relationships between meteorological variables and malaria [19-46]. Except for a single study that found no association, all studies showed correlations between climatic variables and malaria in different locations and study periods in China. The contradictory study likely resulted from a short (6-month) study period [36]. In Yunnan province, two studies were conducted in 2009 to clarify 
Table 2 Characteristics of studies on the association between climatic variables and malaria transmission

\begin{tabular}{|c|c|c|c|c|c|c|}
\hline \multirow{2}{*}{$\begin{array}{l}\text { Study \& } \\
\text { Language }\end{array}$} & \multirow{2}{*}{$\begin{array}{l}\text { Study area \& } \\
\text { period }\end{array}$} & \multicolumn{2}{|l|}{ Data Collection } & \multirow{2}{*}{$\begin{array}{l}\text { Statistical } \\
\text { Methods }\end{array}$} & \multirow[t]{2}{*}{ Main findings } & \multirow[t]{2}{*}{ Comments } \\
\hline & & Risk factors & Disease/vector & & & \\
\hline $\begin{array}{l}\text { Huang et al. } \\
\text { (2011) English } \\
{[19]}\end{array}$ & $\begin{array}{l}\text { Anhui, Henan, } \\
\text { Hubei Provinces } \\
\text { 1990-2009 }\end{array}$ & $\begin{array}{l}\text { Normalized annual } \\
\text { temperature, relative } \\
\text { humidity and rainfall }\end{array}$ & Cases counts & $\begin{array}{l}\text {-Bayesian Poisson } \\
\text { models } \\
\text { - GIS }\end{array}$ & $\begin{array}{l}\text {-Rainfall played a } \\
\text { more important role } \\
\text { in malaria } \\
\text { transmission than } \\
\text { other meteorological } \\
\text { factors. }\end{array}$ & $\begin{array}{l}\text {-Spatial-temporal } \\
\text { models were } \\
\text { developed } \\
\text {-Socioeconomic } \\
\text { factors were not } \\
\text { taken into } \\
\text { account. }\end{array}$ \\
\hline $\begin{array}{l}\text { Huang et al. } \\
\text { (2011) English } \\
\text { [20] }\end{array}$ & $\begin{array}{l}\text { Motuo County, } \\
\text { Tibet 1986-2009 }\end{array}$ & $\begin{array}{l}\text { Monthly average } \\
\text { temperature, } \\
\text { maximum } \\
\text { temperature, } \\
\text { minimum } \\
\text { temperature, relative } \\
\text { humidity and total } \\
\text { amount of rainfall }\end{array}$ & $\begin{array}{l}\text { Monthly incidence } \\
\text { of malaria }\end{array}$ & $\begin{array}{l}\text {-Spearman } \\
\text { correlation } \\
\text { analysis } \\
\text {-Cross-correlation } \\
\text { analysis } \\
\text {-SARIMA model } \\
\text {-Inter-annual } \\
\text { analysis }\end{array}$ & $\begin{array}{l}\text {-Relative humidity } \\
\text { was more sensitive to } \\
\text { monthly malaria } \\
\text { incidence. } \\
\text {-The relationship } \\
\text { between malaria } \\
\text { incidence and rainfall } \\
\text { was not directly and } \\
\text { linearly. }\end{array}$ & $\begin{array}{l}\text {-Several statistical } \\
\text { methods were } \\
\text { applied } \\
\text {-Only one county } \\
\text { was considered }\end{array}$ \\
\hline $\begin{array}{l}\text { Zhou et al. } \\
\text { (2010) English } \\
{[21]}\end{array}$ & $\begin{array}{l}\text { Huaiyuan County } \\
\text { of Anhui and } \\
\text { Tongbai County } \\
\text { of Henan Province } \\
\text { 1990-2006 }\end{array}$ & $\begin{array}{l}\text { Monthly and annual } \\
\text { average temperature, } \\
\text { maximum } \\
\text { temperature, } \\
\text { minimum } \\
\text { temperature, relative } \\
\text { humidity and rainfall }\end{array}$ & $\begin{array}{l}\text { Monthly and } \\
\text { annual incidence } \\
\text { of malaria } \\
\text { Vectorial capacity }\end{array}$ & $\begin{array}{l}\text {-Spearman } \\
\text { correlation } \\
\text {-Stepwise } \\
\text { regression analysis } \\
\text {-Curve fitting } \\
\text {-Trend analysis } \\
\text { - Entomological } \\
\text { investigation }\end{array}$ & $\begin{array}{l}\text {-Temperature and } \\
\text { rainfall were major } \\
\text { determinants for } \\
\text { malaria transmission. } \\
\text { However, no } \\
\text { relationship between } \\
\text { malaria incidence and } \\
\text { relative humidity was } \\
\text { observed. }\end{array}$ & $\begin{array}{l}\text {-Entomological } \\
\text { investigate was } \\
\text { conducted to } \\
\text { determine the } \\
\text { vectorial effect of } \\
\text { malaria re- } \\
\text { emergency. } \\
\text {-Only two } \\
\text { counties were } \\
\text { examined }\end{array}$ \\
\hline $\begin{array}{l}\text { Zhang et al. } \\
\text { (2010) English } \\
{[22]}\end{array}$ & $\begin{array}{l}\text { Jinan city, } \\
\text { Shangdong } \\
\text { Province 1959- } \\
1979\end{array}$ & $\begin{array}{l}\text { Monthly average } \\
\text { maximum } \\
\text { temperature, } \\
\text { minimum } \\
\text { temperature, relative } \\
\text { humidity and rainfall }\end{array}$ & Cases counts & $\begin{array}{l}\text {-Spearman } \\
\text { correlation } \\
\text {-Cross-correlation } \\
\text {-SARIMA model }\end{array}$ & $\begin{array}{l}\text {-Temperature was } \\
\text { greatest relative to } \\
\text { the transmission of } \\
\text { malaria, but rainfall } \\
\text { and relative humidity } \\
\text { were not. }\end{array}$ & $\begin{array}{l}\text {-Only one city was } \\
\text { included } \\
\text {-Socioeconomic } \\
\text { factors ware } \\
\text { ignored. }\end{array}$ \\
\hline $\begin{array}{l}\text { Yang et al. } \\
\text { (2010) English } \\
\text { [23] }\end{array}$ & $\begin{array}{l}\text { The P.R. China } \\
\text { 1981-1995 }\end{array}$ & $\begin{array}{l}\text { Yearly growing } \\
\text { degree days (YGDD), } \\
\text { annual rainfall and } \\
\text { relative humidity }\end{array}$ & $\begin{array}{l}\text { Malaria-endemic } \\
\text { strata }\end{array}$ & $\begin{array}{l}\text {-A Delphi } \\
\text { approach } \\
\text {-Multiple logistical } \\
\text { regression } \\
\text {-GIS }\end{array}$ & $\begin{array}{l}\text {-Relative humidity } \\
\text { was found to be the } \\
\text { most important } \\
\text { environmental factor, } \\
\text { followed by } \\
\text { temperature and } \\
\text { rainfall. However, } \\
\text { temperature was the } \\
\text { major contributor of } \\
\text { malaria intensity in } \\
\text { regions with relative } \\
\text { humidity }>60 \% \text {, }\end{array}$ & $\begin{array}{l}\text {-National-level } \\
\text { analysis } \\
\text {-Risk maps of } \\
\text { malaria based on } \\
\text { different climatic } \\
\text { factors were } \\
\text { developed } \\
\text {-Annual indicators } \\
\text { were used }\end{array}$ \\
\hline $\begin{array}{l}\text { Xiao et al. } \\
\text { (2010) English } \\
{[24]}\end{array}$ & $\begin{array}{l}\text { Main island of } \\
\text { Hainan province } \\
\text { 1995-2008 }\end{array}$ & $\begin{array}{l}\text { Monthly average } \\
\text { temperature, } \\
\text { maximum } \\
\text { temperature, } \\
\text { minimum } \\
\text { temperature, relative } \\
\text { humidity and } \\
\text { accumulative rainfall }\end{array}$ & $\begin{array}{l}\text { Monthly incidence } \\
\text { of malaria }\end{array}$ & $\begin{array}{l}\text {-Cross correlation } \\
\text { and } \\
\text { autocorrelation } \\
\text { analysis } \\
\text {-Poission } \\
\text { regression } \\
\text {-GIS }\end{array}$ & $\begin{array}{l}\text { - Temperature during } \\
\text { the previous one and } \\
\text { two months were } \\
\text { observed as major } \\
\text { predictors of malaria } \\
\text { epidemics. } \\
\text {-lt was not necessary } \\
\text { to consider rainfall } \\
\text { and relative humidity } \\
\text { to make malaria } \\
\text { epidemic predictions } \\
\text { in the tropical } \\
\text { province. }\end{array}$ & $\begin{array}{l}\text {-Countermeasure } \\
\text { and } \\
\text { socioeconomic } \\
\text { circumstances } \\
\text { ware not taken } \\
\text { into account. }\end{array}$ \\
\hline $\begin{array}{l}\text { Hui et al. } \\
\text { (2009) English } \\
{[25]}\end{array}$ & $\begin{array}{l}\text { Yunnan Province } \\
1995-2005\end{array}$ & $\begin{array}{l}\text { Monthly average } \\
\text { temperature, } \\
\text { maximum }\end{array}$ & $\begin{array}{l}\text { Monthly incidence } \\
\text { of } P \text {. vivax malaria } \\
\text { Monthly incidence }\end{array}$ & $\begin{array}{l}\text {-Spearman } \\
\text { correlation } \\
\text { analysis }\end{array}$ & $\begin{array}{l}\text {-Obvious associations } \\
\text { between both } P \text {. vivax } \\
\text { and } P \text {. falciparum }\end{array}$ & $\begin{array}{l}\text {-Analysis of both } \\
P . \text { vivax malaria }\end{array}$ \\
\hline
\end{tabular}


Table 2 Characteristics of studies on the association between climatic variables and malaria transmission (Continued)

\begin{tabular}{|c|c|c|c|c|c|c|}
\hline & & $\begin{array}{l}\text { temperature, } \\
\text { minimum } \\
\text { temperature, relative } \\
\text { humidity and rainfall }\end{array}$ & $\begin{array}{l}\text { of P. falciparum } \\
\text { malaria }\end{array}$ & $\begin{array}{l}\text {-Temporal } \\
\text { distribute analysis } \\
\text {-Spatial } \\
\text { autocorrelation } \\
\text {-Spatial cluster } \\
\text { analysis } \\
\text { - GIS }\end{array}$ & $\begin{array}{l}\text { malaria and climatic } \\
\text { factors with a clear } 1 \text { - } \\
\text { month lagged effect, } \\
\text { especially in cluster } \\
\text { areas. } \\
\text {-Minimum } \\
\text { temperature was } \\
\text { most closely } \\
\text { correlated to malaria } \\
\text { incidence }\end{array}$ & $\begin{array}{l}\text { and } P \text {. falciparum } \\
\text { malaria } \\
\text {-Spatio-temporal } \\
\text { analysis }\end{array}$ \\
\hline $\begin{array}{l}\text { Clements et al. } \\
\text { (2009) English } \\
\text { [26] }\end{array}$ & $\begin{array}{l}\text { Yunnan Province } \\
\text { 1991-2006 }\end{array}$ & $\begin{array}{l}\text { Monthly average } \\
\text { rainfall, maximum } \\
\text { temperature and } \\
\text { minimum } \\
\text { temperature }\end{array}$ & $\begin{array}{l}\text { Monthly incidence } \\
\text { of } P \text {. vivax malaria } \\
\text { Monthly incidence } \\
\text { of } P \text {. falciparum } \\
\text { malaria }\end{array}$ & $\begin{array}{l}\text {-Corss-correlation } \\
\text {-Bayesian Poisson } \\
\text { regression } \\
\text {-GIS }\end{array}$ & $\begin{array}{l}\text {-Significant positive } \\
\text { relationships between } \\
\text { malaria incidence and } \\
\text { rainfall and maximum } \\
\text { temperature for both } \\
P \text {. vivax and } P \text {. } \\
\text { falciparum malaria } \\
\text {-High-incidence } \\
\text { clusters located } \\
\text { adjacent the } \\
\text { international borders } \\
\text { were not explained } \\
\text { by climate, but partly } \\
\text { due to population } \\
\text { migration. }\end{array}$ & $\begin{array}{l}\text {-Analysis of both } \\
\text { P. vivax malaria } \\
\text { and P. falciparum } \\
\text { malaria } \\
\text {-Spatial-temporal } \\
\text { analysis } \\
\text {-Socioeconomic } \\
\text { factors were } \\
\text { ignored. }\end{array}$ \\
\hline $\begin{array}{l}\text { Tian et al. } \\
\text { (2008) English } \\
\text { [27] }\end{array}$ & $\begin{array}{l}\text { Mengla County, } \\
\text { Yunnan Province } \\
\text { 1971-1999 }\end{array}$ & $\begin{array}{l}\text { Monthly rainfall, } \\
\text { minimum } \\
\text { temperature, } \\
\text { maximum } \\
\text { temperature, relative } \\
\text { humidity, and fog day } \\
\text { frequency }\end{array}$ & $\begin{array}{l}\text { Monthly incidence } \\
\text { of malaria }\end{array}$ & -ARIMA models & $\begin{array}{l}\text {-Temperature and fog } \\
\text { day frequency were } \\
\text { key predictors of } \\
\text { monthly malaria } \\
\text { incidence. However, } \\
\text { relative humidity and } \\
\text { rainfall were not. } \\
\text {-The annual fog } \\
\text { frequency was the } \\
\text { only weather } \\
\text { predictor of the } \\
\text { annual incidence of } \\
\text { malaria }\end{array}$ & $\begin{array}{l}\text {-Fog day } \\
\text { frequency used } \\
\text {-P. vivax malaria } \\
\text { and } P \text {. falciparum } \\
\text { malaria were } \\
\text { pooled together } \\
\text { when malaria } \\
\text { incidence was } \\
\text { calculated. }\end{array}$ \\
\hline $\begin{array}{l}\text { Bi et al. (2005) } \\
\text { English [28] }\end{array}$ & $\begin{array}{l}\text { Anhui province } \\
\text { 1966-1987 }\end{array}$ & $\begin{array}{l}\text { Monthly El-Nino } \\
\text { Southern Oscillation } \\
\text { Index (ENSO) }\end{array}$ & $\begin{array}{l}\text { Monthly malaria } \\
\text { cases }\end{array}$ & $\begin{array}{l}\text {-Spearman } \\
\text { correlation }\end{array}$ & $\begin{array}{l}\text {-A positive correlation } \\
\text { between ENSO and } \\
\text { the incidence of } \\
\text { malaria with no lag } \\
\text { effect was found. }\end{array}$ & $\begin{array}{l}\text {-The impact of } \\
\text { ENSO on malaria } \\
\text { was analysed } \\
\text {-Other } \\
\text { meteorological } \\
\text { variables were not } \\
\text { considered. } \\
\text {-Only used } \\
\text { correlation } \\
\text { method }\end{array}$ \\
\hline $\begin{array}{l}\text { Liu et al. } \\
\text { (2006) English } \\
\text { [29] }\end{array}$ & $\begin{array}{l}\text { Twenty-one } \\
\text { townships of } 10 \\
\text { counties in } \\
\text { Yunnan province } \\
\text { 1984-1993 }\end{array}$ & $\begin{array}{l}\text { Monthly minimum } \\
\text { temperature, } \\
\text { maximum } \\
\text { temperature, rainfall, } \\
\text { sunshine duration, } \\
\text { NDVI. }\end{array}$ & $\begin{array}{l}\text { Monthly incidence } \\
\text { of malaria and } \\
\text { vector density. }\end{array}$ & $\begin{array}{l}\text {-Principle } \\
\text { component } \\
\text { analysis } \\
\text {-Factor analysis } \\
\text {-Grey correlation } \\
\text { analysis }\end{array}$ & $\begin{array}{l}\text {-Remote sensing NDVI } \\
\text { and climatic variables } \\
\text { had a good } \\
\text { correlation with } \\
\text { Anopheles density and } \\
\text { malaria incidence rate. }\end{array}$ & $\begin{array}{l}\text {-Both } \\
\text { environmental } \\
\text { and vector factors } \\
\text { were analysed. }\end{array}$ \\
\hline $\begin{array}{l}\text { Bi et al. (2003) } \\
\text { English [30] }\end{array}$ & $\begin{array}{l}\text { Sunchen County } \\
\text { in Ahui Province } \\
\text { 1980-1991 }\end{array}$ & $\begin{array}{l}\text { Monthly maximum } \\
\text { temperature, } \\
\text { minimum } \\
\text { temperature, relative } \\
\text { humidity and rainfall }\end{array}$ & $\begin{array}{l}\text { Monthly incidence } \\
\text { of malaria }\end{array}$ & $\begin{array}{l}\text {-Spearman } \\
\text { correlation } \\
\text {-Cross-correlation } \\
\text {-ARIMA models }\end{array}$ & $\begin{array}{l}\text {-Monthly average } \\
\text { minimum } \\
\text { temperature and total } \\
\text { monthly rainfall, at } \\
\text { one-month lag were } \\
\text { major determinants in } \\
\text { the transmission of } \\
\text { malaria. }\end{array}$ & $\begin{array}{l}\text {-Non-climatic } \\
\text { factors were } \\
\text { neglected } \\
\text {-Only one county } \\
\text { considered }\end{array}$ \\
\hline
\end{tabular}


Table 2 Characteristics of studies on the association between climatic variables and malaria transmission (Continued)

\begin{tabular}{|c|c|c|c|c|c|c|}
\hline $\begin{array}{l}\text { Hu et al. } \\
\text { (1998) English } \\
{[31]}\end{array}$ & $\begin{array}{l}\text { Yunnan Province } \\
\text { 1991-1997 }\end{array}$ & $\begin{array}{l}\text { Annual rainfall, annual } \\
\text { mean temperature }\end{array}$ & $\begin{array}{l}\text { Annual incidence } \\
\text { of malaria }\end{array}$ & $\begin{array}{l}\text { - Multiple } \\
\text { regression } \\
\text {-GIS }\end{array}$ & $\begin{array}{l}\text {-Malaria incidence } \\
\text { rates are higher in } \\
\text { areas with } \\
\text { temperature above } \\
18^{\circ} \mathrm{C} \text {, rainfall of more } \\
\text { than } 1000 \mathrm{~mm}\end{array}$ & $\begin{array}{l}\text {-Socioeconomic } \\
\text { factors such as } \\
\text { income of farmers } \\
\text { were taken into } \\
\text { account. }\end{array}$ \\
\hline & & & & & $\begin{array}{l}\text {-Every one degree } \\
\text { increase in } \\
\text { temperature } \\
\text { corresponds to 1.2/ } \\
10,000 \text { higher malaria } \\
\text { incidence and when } \\
\text { rainfall increase by } \\
100 \text { mm, malaria will } \\
\text { increase to 100.0/ } \\
10,000\end{array}$ & $\begin{array}{l}\text {-Annual data were } \\
\text { used }\end{array}$ \\
\hline $\begin{array}{l}\text { Liu et al. } \\
\text { (2011) Chinese } \\
\text { [32] }\end{array}$ & $\begin{array}{l}\text { Pizhou City, } \\
\text { Jiangsu province } \\
2001-2006\end{array}$ & $\begin{array}{l}\text { Monthly mean } \\
\text { temperature, } \\
\text { maximum } \\
\text { temperature, } \\
\text { minimum } \\
\text { temperature, rainfall } \\
\text { days, relative } \\
\text { humidity, evaporation, } \\
\text { total cloud cover, } \\
\text { sunlight time and low } \\
\text { cloud. }\end{array}$ & $\begin{array}{l}\text { Monthly incidence } \\
\text { of malaria }\end{array}$ & $\begin{array}{l}\text {-Correlation } \\
\text { analysis } \\
\text {-Multiple } \\
\text { regression }\end{array}$ & $\begin{array}{l}\text {-The incidence of } \\
\text { malaria was passive } \\
\text { relative to } \\
\text { temperature, rainfall, } \\
\text { relative humidity, } \\
\text { evaporation and total } \\
\text { cloud cover, but no } \\
\text { relation with low } \\
\text { cloud and sunlight. } \\
\text {-The monthly } \\
\text { minimum } \\
\text { temperature and } \\
\text { relative humidity were } \\
\text { two major factors } \\
\text { influencing malaria } \\
\text { transmission. }\end{array}$ & $\begin{array}{l}\text {-Various } \\
\text { meteorological } \\
\text { variables were } \\
\text { considered } \\
\text {-Only one city was } \\
\text { analysed based on } \\
\text { a relative short } \\
\text { study period }\end{array}$ \\
\hline $\begin{array}{l}\text { Wu et al. } \\
\text { (2011) Chinese } \\
{[33]}\end{array}$ & $\begin{array}{l}\text { Dianjiang county, } \\
\text { Chongqing 1957- } \\
2010\end{array}$ & $\begin{array}{l}\text { Monthly mean } \\
\text { temperature, } \\
\text { maximum } \\
\text { temperature, } \\
\text { minimum } \\
\text { temperature, rainfall } \\
\text { days, relative } \\
\text { humidity, absolute } \\
\text { humidity, duration of } \\
\text { sunshine, air pressure } \\
\text { and wind speed. }\end{array}$ & Case counts & $\begin{array}{l}\text {-Principal } \\
\text { Component } \\
\text { Analysis } \\
\text {-Multiple } \\
\text { regression }\end{array}$ & $\begin{array}{l}\text {-Significant } \\
\text { associations between } \\
\text { malaria incidence and } \\
\text { monthly mean } \\
\text { temperature, rainfall } \\
\text { and duration of } \\
\text { sunshine were } \\
\text { observed. } \\
\text {-Temperature was } \\
\text { greatest relative to } \\
\text { malaria transmission }\end{array}$ & $\begin{array}{l}\text {-Various } \\
\text { meteorological } \\
\text { variables were } \\
\text { considered } \\
\text {-Long-term data } \\
\text { from a fifty-four } \\
\text {-years period } \\
\text {-Only one county } \\
\text { considered }\end{array}$ \\
\hline $\begin{array}{l}\text { Huang et al. } \\
\text { (2009) Chinese } \\
\text { [34] }\end{array}$ & $\begin{array}{l}\text { Tongbai and } \\
\text { Dabie mountain } \\
\text { areas, Huibei } \\
\text { Province 1990- } \\
2007\end{array}$ & $\begin{array}{l}\text { Monthly mean } \\
\text { temperature, } \\
\text { maximum } \\
\text { temperature, } \\
\text { minimum } \\
\text { temperature, rainfall. }\end{array}$ & Case counts & Descriptive study & $\begin{array}{l}\text {-Temperature and } \\
\text { rainfall were major } \\
\text { determinants for } \\
\text { malaria transmission } \\
\text { and the yearly peak of } \\
\text { cases occurred one } \\
\text { month after the rainy } \\
\text { season. }\end{array}$ & $\begin{array}{l}\text {-Not enough } \\
\text { statistical methods }\end{array}$ \\
\hline $\begin{array}{l}\text { Wang et al. } \\
\text { (2009) Chinese } \\
\text { [35] }\end{array}$ & $\begin{array}{l}\text { Anhui Province } \\
\text { 2004-2006 }\end{array}$ & $\begin{array}{l}\text { Annually mean } \\
\text { temperature and } \\
\text { rainfall NDVI and } \\
\text { elevation. }\end{array}$ & Cases counts & $\begin{array}{l}\text {-Principal } \\
\text { Component } \\
\text { Analysis } \\
\text {-Logistic } \\
\text { regression } \\
\text {-GIS }\end{array}$ & $\begin{array}{l}\text {-Malaria transmission } \\
\text { intensity was } \\
\text { positively associated } \\
\text { with the NDVI, but } \\
\text { negatively associated } \\
\text { with minimum } \\
\text { temperature, rainfall } \\
\text { and elevation. }\end{array}$ & $\begin{array}{l}\text {-Annual indicators } \\
\text { were used } \\
\text {-A two-years short } \\
\text { period of study. }\end{array}$ \\
\hline $\begin{array}{l}\text { Wen et al. } \\
\text { (2008) Chinese } \\
{[36]}\end{array}$ & $\begin{array}{l}\text { Hainan Province } \\
\text { May-Oct in } 2002\end{array}$ & $\begin{array}{l}\text { Monthly mean } \\
\text { temperature, } \\
\text { maximum } \\
\text { temperature, } \\
\text { minimum }\end{array}$ & $\begin{array}{l}\text { Monthly incidence } \\
\text { of malaria }\end{array}$ & $\begin{array}{l}\text {-Spearman } \\
\text { correlation }\end{array}$ & $\begin{array}{l}\text {-No associations } \\
\text { between } \\
\text { meteorological factors } \\
\text { and malaria incidence } \\
\text { were observed. }\end{array}$ & $\begin{array}{l}\text {-Various } \\
\text { environmental } \\
\text { variables were } \\
\text { collected }\end{array}$ \\
\hline
\end{tabular}


Table 2 Characteristics of studies on the association between climatic variables and malaria transmission (Continued)

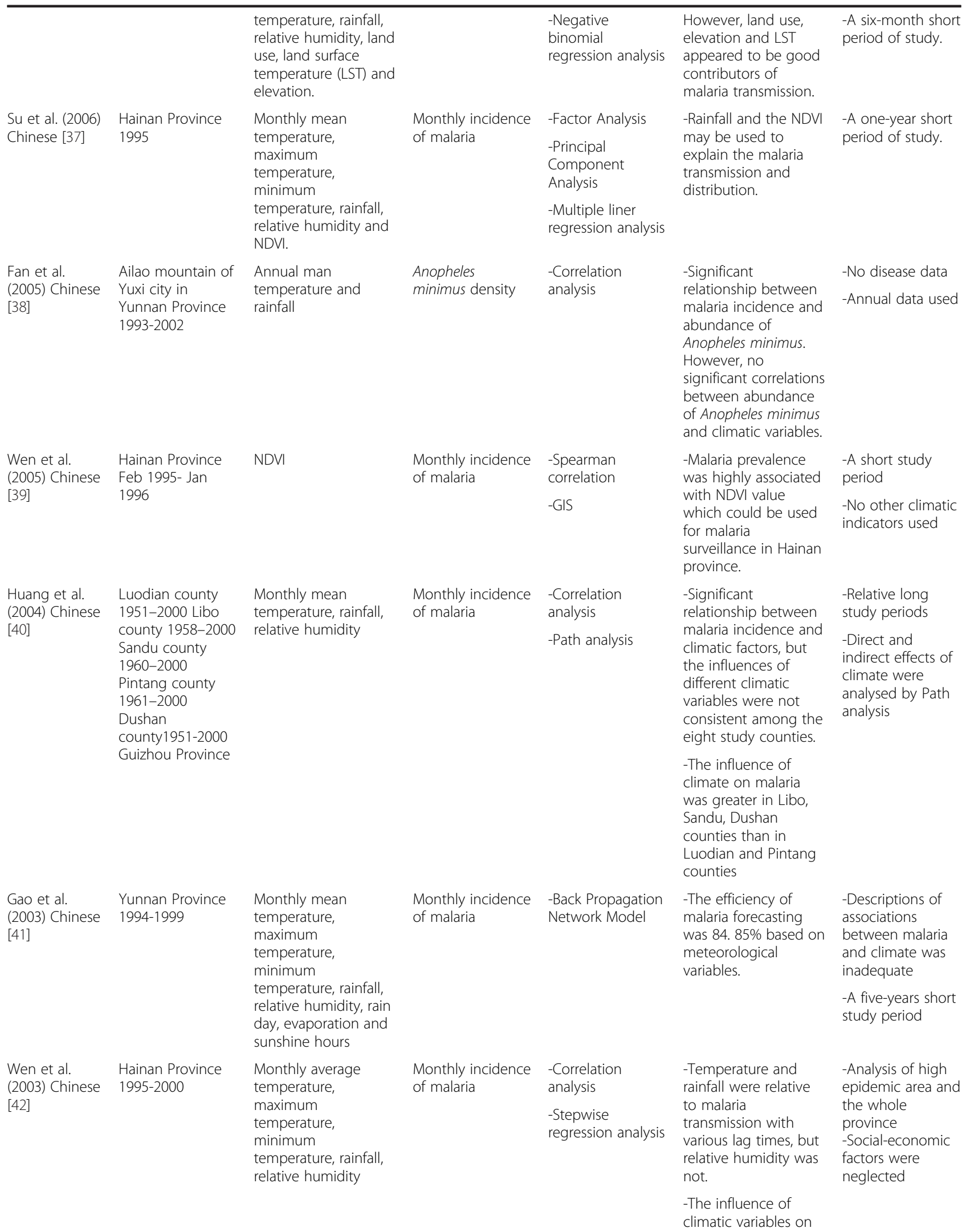




\begin{tabular}{|c|c|c|c|c|c|c|}
\hline & & & & & $\begin{array}{l}\text { malaria was more } \\
\text { obvious in high } \\
\text { epidemic area than } \\
\text { that in the whole } \\
\text { province }\end{array}$ & \\
\hline $\begin{array}{l}\text { Huang et al. } \\
\text { (2002) Chinese } \\
\text { [43] }\end{array}$ & $\begin{array}{l}\text { Jiangsu Province } \\
\text { 1973-1983 }\end{array}$ & $\begin{array}{l}\text { Monthly rainfall, rain } \\
\text { days, relative } \\
\text { humidity, evaporation } \\
\text { and NDVI }\end{array}$ & $\begin{array}{l}\text { Monthly incidence } \\
\text { of malaria }\end{array}$ & $\begin{array}{l}\text {-Correlation } \\
\text { analysis } \\
\text {-GIS }\end{array}$ & $\begin{array}{l}\text {-The NDVI positively } \\
\text { correlated with } \\
\text { precipitation and } \\
\text { relative humidity. } \\
\text {-The NDVI may be a } \\
\text { good indicator to } \\
\text { predict the } \\
\text { distribution and } \\
\text { transmission of } \\
\text { malaria. }\end{array}$ & $\begin{array}{l}\text {-No temperature } \\
\text { data included } \\
\text {-Only correlation } \\
\text { method used }\end{array}$ \\
\hline $\begin{array}{l}\text { Huang et al. } \\
\text { (2001) Chinese } \\
{[44]}\end{array}$ & $\begin{array}{l}\text { Gaoan city, Jiangxi } \\
\text { Province 1962- } \\
1999\end{array}$ & $\begin{array}{l}\text { Annually average } \\
\text { rainfall during April to } \\
\text { June, annually } \\
\text { average temperature } \\
\text { during July to August, } \\
\text { annual average } \\
\text { rainfall and } \\
\text { temperature }\end{array}$ & Case counts & $\begin{array}{l}\text {-Circular } \\
\text { distribution } \\
\text { method } \\
\text {-Descriptive study }\end{array}$ & $\begin{array}{l}\text {-Malaria cases } \\
\text { increased with } \\
\text { increase of average } \\
\text { temperature from July } \\
\text { to August and rainfall } \\
\text { from April to June. }\end{array}$ & $\begin{array}{l}\text {-Annual index } \\
\text { were used }\end{array}$ \\
\hline $\begin{array}{l}\text { Kan et al. } \\
\text { (1999) Chinese } \\
{[45]}\end{array}$ & $\begin{array}{l}\text { Anhui Province } \\
\text { 1969-1999 }\end{array}$ & $\begin{array}{l}\text { Annual temperature } \\
\text { and rainfall }\end{array}$ & $\begin{array}{l}\text { Annual incidence } \\
\text { of malaria }\end{array}$ & -Descriptive study & $\begin{array}{l}\text {-Annual incidences of } \\
\text { malaria in 1975, 1977, } \\
1980 \text { in Madian, Lixin } \\
\text { County increased } \\
\text { with increase of } \\
\text { rainfall, while } \\
\text { decreased in 1976, } \\
\text { 1978, } 1981 \text { with } \\
\text { decreased rainfall }\end{array}$ & $\begin{array}{l}\text {-Not enough } \\
\text { explanation on } \\
\text { effects of climate } \\
\text { factors on malaria. } \\
\text {-No statistical } \\
\text { methods used }\end{array}$ \\
\hline $\begin{array}{l}\text { Yu et al. (1995) } \\
\text { Chinese [46] }\end{array}$ & $\begin{array}{l}\text { Libo County, } \\
\text { Guizhou Province } \\
\text { 1958-1993 }\end{array}$ & $\begin{array}{l}\text { Monthly average } \\
\text { temperature, rainfall, } \\
\text { relative humidity }\end{array}$ & $\begin{array}{l}\text { Monthly incidence } \\
\text { of malaria }\end{array}$ & $\begin{array}{l}\text {-Correlation } \\
\text { analysis } \\
\text {-Path analysis }\end{array}$ & $\begin{array}{l}\text {-Positive associations } \\
\text { between malaria } \\
\text { incidence and } \\
\text { climatic factors were } \\
\text { observed. } \\
\text {-Direct effect of } \\
\text { relative humidity was } \\
\text { greatest on malaria } \\
\text { incidence compared } \\
\text { with temperature and } \\
\text { rainfall. }\end{array}$ & $\begin{array}{l}\text {-Relative long } \\
\text { study periods } \\
\text {-Direct and } \\
\text { indirect effects of } \\
\text { climate were } \\
\text { analysed }\end{array}$ \\
\hline
\end{tabular}

potential risk factors of malaria transmission [26,31]. Clements et al. (2009) demonstrated that for P. vivax the relative risk appeared to cycle every 3 to 4 years, whereas for $P$. falciparum the pattern was less regular [26]. Hui et al. (2009) found that the influence of meteorological variables on $P$. vivax malaria was greater than that of $P$. falciparum malaria, especially in cluster areas, indicating $P$. vivax malaria may be more climatesensitive [31].

Almost all of these analyses identified a positive association between temperature indices and malaria transmission. Some studies also reported that temperature was the most important climatic determinant in the transmission of malaria. For example, a study conducted in
Jinan, which is a temperate city in northern China, showed that a $1^{\circ} \mathrm{C}$ rise in maximum temperature may be related to a $7.7 \%$ to $12.7 \%$ increase in the number of malaria cases, while a $1^{\circ} \mathrm{C}$ rise in minimum temperature may result in approximately $11.8 \%$ to $12.7 \%$ increase in cases [22]. Zhou et al. (2010) revealed that temperature was a key meteorological factor correlated to malaria incidence, implying the potential role of global warming in malaria re-emergence in central China early in the 21th century, especially in Anhui, Henan and Hubei Provinces along the Huang-Huai River [21]. However, the association between temperature variables and malaria incidence may not be constant year-round. Tian et al. (2008) emphasized the stronger effect of minimum temperature on malaria 
Table 3 Characteristics of studies on the association between climatic variables and dengue transmission

\begin{tabular}{|c|c|c|c|c|c|c|}
\hline \multirow{2}{*}{$\begin{array}{l}\text { Study \& } \\
\text { Language }\end{array}$} & \multirow{2}{*}{$\begin{array}{l}\text { Study area \& } \\
\text { period }\end{array}$} & \multicolumn{2}{|l|}{ Data Collection } & \multirow{2}{*}{$\begin{array}{l}\text { Statistical } \\
\text { methods }\end{array}$} & \multirow[t]{2}{*}{ Main findings } & \multirow[t]{2}{*}{ Comments } \\
\hline & & Risk factors & Disease/vector & & & \\
\hline \multirow{2}{*}{$\begin{array}{l}\text { Wu et al. } \\
\text { (2011) English } \\
\text { [47] }\end{array}$} & \multirow{2}{*}{$\begin{array}{l}\text { Liaoning, Hebei, } \\
\text { Shanxi, Shaanxi, } \\
\text { Sichuan, and } \\
\text { Gansu Province } \\
\text { 1961-1990 }\end{array}$} & \multirow{2}{*}{$\begin{array}{l}\text { Annual temperature } \\
\text { and precipitation, the } \\
\text { monthly temperature } \\
\text { in January }\end{array}$} & \multirow{2}{*}{$\begin{array}{l}\text { Distribution data } \\
\text { of Aedes } \\
\text { albopictus }\end{array}$} & $\begin{array}{l}\text {-CLIMEX } \\
\text { model }\end{array}$ & \multirow{2}{*}{$\begin{array}{l}\text {-Aedes albopictus have } \\
\text { extended their } \\
\text { geographic range to } \\
\text { areas, which } \\
\text { experienced the } \\
\text { annual mean } \\
\text { temperature below } \\
11^{\circ} \mathrm{C} \text { and the January } \\
\text { mean temperature } \\
\text { below }-5^{\circ} \mathrm{Cand} \text { this } \\
\text { may be due to } \\
\text { summer expansion }\end{array}$} & \multirow{2}{*}{$\begin{array}{l}\text {-Risk maps of the } \\
\text { potential } \\
\text { distribution of } \\
\text { Aedes albopictus in } \\
\text { China were } \\
\text { developed } \\
\text { - No disease } \\
\text { variables included }\end{array}$} \\
\hline & & & & $-G I S$ & & \\
\hline \multirow[t]{3}{*}{$\begin{array}{l}\text { Lai et al. } \\
\text { (2011) English } \\
\text { [48] }\end{array}$} & \multirow[t]{3}{*}{$\begin{array}{l}\text { Kaohsiung City, } \\
\text { Taiwan 2002-2007 }\end{array}$} & \multirow{3}{*}{$\begin{array}{l}\text { Daily air temperature, } \\
\text { amount of rainfall, } \\
\text { relative humidity, sea } \\
\text { surface temperature } \\
\text { (SST) and weather } \\
\text { patterns of typhoons }\end{array}$} & \multirow{3}{*}{$\begin{array}{l}\text { Daily number of } \\
\text { hospital } \\
\text { admissions for } \\
\text { dengue fever The } \\
\text { incidence of } \\
\text { dengue fever, } \\
\text { Breteau Index }\end{array}$} & $\begin{array}{l}\text {-Cross- } \\
\text { correlation }\end{array}$ & \multirow{3}{*}{$\begin{array}{l}\text {-Hospital admissions } \\
\text { for dengue in } 2002 \\
\text { and } 2005 \text { were } \\
\text { correlated with } \\
\text { climatic factors with } \\
\text { different time lags, } \\
\text { including } \\
\text { precipitation, } \\
\text { temperature and the } \\
\text { minimum relative } \\
\text { humidity }\end{array}$} & $\begin{array}{l}\text {-Both disease and } \\
\text { vector factors } \\
\text { were considered. }\end{array}$ \\
\hline & & & & $\begin{array}{l}\text {-Duncan's } \\
\text { Multiple } \\
\text { Range test }\end{array}$ & & $\begin{array}{l}\text {-The impacts of } \\
\text { SST and typhoons } \\
\text { were discussed. }\end{array}$ \\
\hline & & & & $\begin{array}{l}\text {-Spatial auto- } \\
\text { correlation } \\
\text { analysis }\end{array}$ & & $\begin{array}{l}\text {-Two case studies } \\
\text { of dengue events } \\
\text { were included. }\end{array}$ \\
\hline
\end{tabular}

\begin{tabular}{|c|c|c|c|c|c|c|}
\hline & & & & -GIS & $\begin{array}{l}\text {-Warm sea surface } \\
\text { temperature and } \\
\text { weather pattern of } \\
\text { typhoons were major } \\
\text { contributor to } \\
\text { outbreaks of dengue }\end{array}$ & \\
\hline \multirow[t]{3}{*}{$\begin{array}{l}\text { Chen et al. } \\
\text { (2010) English } \\
\text { [49] }\end{array}$} & \multirow[t]{3}{*}{$\begin{array}{l}\text { Taipei and } \\
\text { Kaohsiung, Taiwan } \\
\text { 2001-2008 }\end{array}$} & \multirow{3}{*}{$\begin{array}{l}\text { Weekly minimum, } \\
\text { mean, and maximum } \\
\text { temperatures, relative } \\
\text { humidity and rainfall }\end{array}$} & \multirow[t]{3}{*}{$\begin{array}{l}\text { Weekly dengue } \\
\text { incidence Breteau } \\
\text { Index }\end{array}$} & $\begin{array}{l}\text {-Poisson } \\
\text { regression } \\
\text { analysis }\end{array}$ & \multirow{2}{*}{$\begin{array}{l}\text {-Weak positive } \\
\text { relationships between } \\
\text { dengue incidence } \\
\text { and temperature } \\
\text { variables in Taipei } \\
\text { were found, whereas } \\
\text { in Kaohsiung, all } \\
\text { climatic factors were } \\
\text { negatively correlated } \\
\text { with dengue } \\
\text { incidence }\end{array}$} & $\begin{array}{l}\text {-Both disease and } \\
\text { vector factors } \\
\text { were considered. }\end{array}$ \\
\hline & & & & $\begin{array}{l}\text {-Spearman } \\
\text { correlation }\end{array}$ & & $\begin{array}{l}\text {-Weekly indicators } \\
\text { were used }\end{array}$ \\
\hline & & & & & $\begin{array}{l}\text {-Climatic factors with } \\
3 \text {-month lag, and 1- } \\
\text { month lag of } \\
\text { percentage Bl level } \\
>2 \text { were the } \\
\text { significant predictors } \\
\text { of dengue incidence } \\
\text { in Kaohsiung }\end{array}$ & \\
\hline \multirow{3}{*}{$\begin{array}{l}\text { Shang et al. } \\
\text { (2010) English } \\
\text { [50] }\end{array}$} & \multirow{3}{*}{$\begin{array}{l}\text { Southern Taiwan } \\
\text { (Tainan, Kaohsiung } \\
\text { and Pingtung) } \\
1998-2007\end{array}$} & \multirow{3}{*}{$\begin{array}{l}\text { Daily mean } \\
\text { temperature, } \\
\text { maximum } \\
\text { temperature, } \\
\text { minimum } \\
\text { temperature, relative } \\
\text { humidity, wind speed, } \\
\text { sunshine } \\
\text { accumulation hours, } \\
\text { sunshine rate, } \\
\text { sunshine total flux } \\
\text { and accumulative } \\
\text { rainfall, accumulative } \\
\text { rainy hours. }\end{array}$} & \multirow{3}{*}{$\begin{array}{l}\text { Indigenous } \\
\text { dengue cases } \\
\text { Imported dengue } \\
\text { cases }\end{array}$} & $\begin{array}{l}\text {-Logistic } \\
\text { regression }\end{array}$ & \multirow{3}{*}{$\begin{array}{l}\text {-An increase in } \\
\text { imported case favors } \\
\text { the occurrence of } \\
\text { indigenous dengue } \\
\text { when warmer and } \\
\text { drier weather } \\
\text { conditions are present }\end{array}$} & \multirow{2}{*}{$\begin{array}{l}\text {-Simultaneously } \\
\text { identify the } \\
\text { relationship } \\
\text { between } \\
\text { indigenous and } \\
\text { imported dengue } \\
\text { cases in the } \\
\text { context of } \\
\text { meteorological } \\
\text { factors }\end{array}$} \\
\hline & & & & $\begin{array}{l}\text {-Poisson } \\
\text { regression }\end{array}$ & & \\
\hline & & & & & & $\begin{array}{l}\text {-Various climatic } \\
\text { data were } \\
\text { considered. }\end{array}$ \\
\hline
\end{tabular}


Table 3 Characteristics of studies on the association between climatic variables and dengue transmission (Continued)

\begin{tabular}{|c|c|c|c|c|c|c|}
\hline $\begin{array}{l}\text { Lu et al. (2009) } \\
\text { English [51] }\end{array}$ & $\begin{array}{l}\text { Guangzhou City, } \\
\text { Guangdong } \\
\text { Province 2001- } \\
2006\end{array}$ & $\begin{array}{l}\text { Monthly minimum } \\
\text { temperature, } \\
\text { maximum } \\
\text { temperature, total } \\
\text { rainfall, minimum } \\
\text { relative humidity,wind } \\
\text { velocity }\end{array}$ & $\begin{array}{l}\text { Monthly dengue } \\
\text { fever cases and } \\
\text { incidences }\end{array}$ & $\begin{array}{l}\text {-Spearman } \\
\text { correlation } \\
\text {-Poisson } \\
\text { regression }\end{array}$ & $\begin{array}{l}\text {-Dengue incidence } \\
\text { was positively } \\
\text { associated with } \\
\text { minimum } \\
\text { temperature and } \\
\text { negatively with wind } \\
\text { velocity. }\end{array}$ & $\begin{array}{l}\text {-A relative short 5- } \\
\text { years study } \\
\text { period. } \\
\text {-Other } \\
\text { environmental } \\
\text { and host factors } \\
\text { were ignored. }\end{array}$ \\
\hline $\begin{array}{l}\text { Hsieh et al. } \\
\text { (2009) English } \\
\text { [52] }\end{array}$ & Taiwan 2007 & $\begin{array}{l}\text { Typhoons, weekly } \\
\text { temperature and total } \\
\text { precipitation }\end{array}$ & $\begin{array}{l}\text { Weekly dengue } \\
\text { incidence Initial } \\
\text { reproduction } \\
\text { numbers for the } \\
\text { multi-wave } \\
\text { outbreaks }\end{array}$ & $\begin{array}{l}\text {-Correlation } \\
\text { analysis } \\
\text {-Multi-phase } \\
\text { Richards } \\
\text { model }\end{array}$ & $\begin{array}{l}\text {-A two-wave } \\
\text { outbreaks with } \\
\text { multiple turning } \\
\text { points in } 2007 \text { were } \\
\text { appeared to be led } \\
\text { by the drastic drop in } \\
\text { temperature and } \\
\text { unusually large rainfall } \\
\text { caused by the two } \\
\text { consecutive typhoons. }\end{array}$ & $\begin{array}{l}\text {-The important } \\
\text { role of } \\
\text { climatological } \\
\text { events in dengue } \\
\text { outbreaks was } \\
\text { evaluated. }\end{array}$ \\
\hline $\begin{array}{l}\text { Yang et al. } \\
\text { (2009) English } \\
\text { [53] }\end{array}$ & $\begin{array}{l}\text { Cixi area, Zhejiang } \\
\text { Province (July- } \\
\text { October, 2004) }\end{array}$ & $\begin{array}{l}\text { Daily average } \\
\text { temperature, rainfall, } \\
\text { relative humidity }\end{array}$ & Case counts & $\begin{array}{l}\text {-Descriptive } \\
\text { analysis }\end{array}$ & $\begin{array}{l}\text {-No relationship } \\
\text { between the } \\
\text { incidence of dengue } \\
\text { and meteorological } \\
\text { factors was observed } \\
\text { during the outbreak } \\
\text { in } 2007\end{array}$ & $\begin{array}{l}\text {-A short 6-months } \\
\text { study period. } \\
\text { - No statistical } \\
\text { methods }\end{array}$ \\
\hline $\begin{array}{l}\text { Wu et al. } \\
(2009) \text { English } \\
{[54]}\end{array}$ & Taiwan 1998-2002 & $\begin{array}{l}\text { Monthly temperature } \\
\text { and rainfall } \\
\text { Urbanization level }\end{array}$ & $\begin{array}{l}\text { Monthly incidence } \\
\text { Bl }\end{array}$ & $\begin{array}{l}\text {-Principle } \\
\text { components } \\
\text { analysis } \\
\text {-Logistic } \\
\text { regression } \\
\text {-GIS }\end{array}$ & $\begin{array}{l}\text {-Numbers of months } \\
\text { with average } \\
\text { temperature higher } \\
\text { than } 18^{\circ} \mathrm{C} \text { and high } \\
\text { degree of } \\
\text { urbanization were } \\
\text { identified as } \\
\text { significant indicators } \\
\text { for dengue fever } \\
\text { infections }\end{array}$ & $\begin{array}{l}\text {-Both climatic } \\
\text { variables and } \\
\text { socioeconomic } \\
\text { factors were } \\
\text { considered. }\end{array}$ \\
\hline $\begin{array}{l}\text { Wu et al. } \\
(2007) \text { English } \\
{[55]}\end{array}$ & $\begin{array}{l}\text { Kaohsiung city, } \\
\text { Taiwan 1998-2003 }\end{array}$ & $\begin{array}{l}\text { Monthly average } \\
\text { temperature, } \\
\text { maximum } \\
\text { temperature, } \\
\text { minimum } \\
\text { temperature, relative } \\
\text { humidity, and amount } \\
\text { of rainfall }\end{array}$ & $\begin{array}{l}\text { Monthly incidence } \\
\text { Vector density }\end{array}$ & $\begin{array}{l}\text {-Cross- } \\
\text { correlation } \\
\text {-Auto- } \\
\text { correlation } \\
\text {-ARIMA } \\
\text { models }\end{array}$ & $\begin{array}{l}\text {-Increased incidence } \\
\text { of dengue fever was } \\
\text { associated with } \\
\text { decreased } \\
\text { temperature and } \\
\text { relative humidity. } \\
\text {-Vector density did } \\
\text { not found to be a } \\
\text { good contributor of } \\
\text { disease occurrences. }\end{array}$ & $\begin{array}{l}\text {-Vector density } \\
\text { was analyzed with } \\
\text { dengue incidence } \\
\text { Only one city was } \\
\text { conducted }\end{array}$ \\
\hline $\begin{array}{l}\text { Lu et al. } 2010 \\
\text { Chinese [56] }\end{array}$ & $\begin{array}{l}\text { The P.R. China } \\
1970-2000 \\
\text { Guangzhou City } \\
\text { and Fujian } \\
\text { Province and } \\
\text { Ningbo City 2004- } \\
2006\end{array}$ & $\begin{array}{l}\text { Weekly average } \\
\text { temperature, } \\
\text { maximum } \\
\text { temperature, } \\
\text { minimum } \\
\text { temperature, relative } \\
\text { humidity, rainfall and } \\
\text { duration of sunshine }\end{array}$ & Case counts & $\begin{array}{l}\text {-Correlation } \\
\text { analysis -GIS }\end{array}$ & $\begin{array}{l}\text {-DF outbreaks were } \\
\text { significantly correlated } \\
\text { with climatic variables } \\
\text { with } 8-10 \text { weeks lags. }\end{array}$ & $\begin{array}{l}\text {-A risk map of DF } \\
\text { outbreaks for } \\
\text { China with } \\
\text { suitable weather } \\
\text { conditions was } \\
\text { developed }\end{array}$ \\
\hline $\begin{array}{l}\text { Yu et al. (2005) } \\
\text { Chinese [57] }\end{array}$ & $\begin{array}{l}\text { Hainan Province } \\
\text { (before1986, } \\
\text { 1986-2001) }\end{array}$ & $\begin{array}{l}\text { Monthly temperature } \\
\text { of January Predicted } \\
\text { temperature of winter } \\
\text { in } 2020,2030 \text { and } \\
2050\end{array}$ & $\begin{array}{l}\text { Infectious life span } \\
\text { of infected } \\
\text { mosquito }\end{array}$ & $\begin{array}{l}\text {-Descriptive } \\
\text { analysis } \\
\text {-GIS } \\
\text {-Calculation of } \\
\text { infectious life } \\
\text { span of } \\
\text { mosquito in } \\
\text { different time } \\
\text { periods }\end{array}$ & $\begin{array}{l}\text {-Based on } \\
\text { assumptions that } \\
\text { temperatures in } \\
\text { winter will increase by } \\
1^{\circ} \mathrm{C} \text { and } 2^{\circ} \mathrm{C} \text { in } 2030 \\
\text { and } 2050 \text { respectively, } \\
\text { half of or more areas } \\
\text { in Hainan Province } \\
\text { may be potentially } \\
\text { favorable for dengue } \\
\text { transmission all the } \\
\text { year around by } 2030 \\
\text { and } 2050 \text {. }\end{array}$ & $\begin{array}{l}\text {-Long-term } \\
\text { temperature data } \\
\text { were collected } \\
\text {-Only considered } \\
\text { the temperature } \\
\text {-No disease data } \\
\text { analysed }\end{array}$ \\
\hline
\end{tabular}


Table 3 Characteristics of studies on the association between climatic variables and dengue transmission (Continued)

\begin{tabular}{|c|c|c|c|c|c|c|}
\hline $\begin{array}{l}\text { Chen et al. } \\
\text { (2003) Chinese } \\
{[58]}\end{array}$ & $\begin{array}{l}\text { Nine cities of } \\
\text { Guangdong } \\
\text { Province (Dec } \\
\text { 2000- Nov 2001) }\end{array}$ & $\begin{array}{l}\text { Monthly mean } \\
\text { temperature, relative } \\
\text { humidity, rainfall and } \\
\text { rainy days }\end{array}$ & $\begin{array}{l}\text { Case counts } \\
\text { Breteau index }\end{array}$ & $\begin{array}{l}\text {-Descriptive } \\
\text { analysis }\end{array}$ & $\begin{array}{l}\text {-The dengue fever } \\
\text { intensity was highly } \\
\text { related to increased } \\
\text { temperature }\left(>26^{\circ} \mathrm{C}\right) \text {, } \\
\text { rainfall and } \\
\text { consecutive rainy } \\
\text { days ( }>10 \text { days). }\end{array}$ & $\begin{array}{l}\text {-Study period was } \\
\text { short } \\
\text {-No statistical } \\
\text { methods }\end{array}$ \\
\hline $\begin{array}{l}\text { Yi et al. (2003) } \\
\text { Chinese [59] }\end{array}$ & $\begin{array}{l}\text { Chaozhou City, } \\
\text { Guangdong } \\
\text { Province 1995- } \\
2001\end{array}$ & $\begin{array}{l}\text { Monthly mean } \\
\text { temperature, } \\
\text { maximum } \\
\text { temperature, } \\
\text { minimum } \\
\text { temperature, relative } \\
\text { humidity, rainfall, rainy } \\
\text { days, duration of } \\
\text { sunshine }\end{array}$ & $\begin{array}{l}\text { Case counts } \\
\text { Breteau index }\end{array}$ & $\begin{array}{l}\text {-Pearson } \\
\text { correlation } \\
\text {-Stepwise } \\
\text { regression } \\
\text {-Logistic } \\
\text { regression }\end{array}$ & $\begin{array}{l}\text {-Aedes density was } \\
\text { positively correlated } \\
\text { with temperature, } \\
\text { rainfall, number of } \\
\text { rainy days, duration of } \\
\text { sunshine and } \\
\text { negatively linked to } \\
\text { relative humidity. } \\
\text {-Minimum } \\
\text { temperature, rainfall } \\
\text { and relative humidity } \\
\text { are good predictors } \\
\text { of Adeds density and } \\
\text { dengue transmission. }\end{array}$ & $\begin{array}{l}\text {-Various } \\
\text { meteorological } \\
\text { variables were } \\
\text { used } \\
\text {-Lag times of } \\
\text { climatic factors } \\
\text { were not analysed } \\
\text {-Both climatic } \\
\text { variables and } \\
\text { vector factors } \\
\text { considered. }\end{array}$ \\
\hline $\begin{array}{l}\text { Chen et al. } \\
\text { (2002) Chinese } \\
{[60]}\end{array}$ & $\begin{array}{l}\text { Hainan Province } \\
\text { 1987-1996 }\end{array}$ & Monthly temperature & $\begin{array}{l}\text { Infectious life span } \\
\text { of infected } \\
\text { mosquito }\end{array}$ & $\begin{array}{l}\text {-Descriptive } \\
\text { analysis } \\
\text {-Calculation of } \\
\text { infectious life } \\
\text { span of } \\
\text { mosquito } \\
\text { under } \\
\text { different } \\
\text { temperature }\end{array}$ & $\begin{array}{l}\text {-If temperature } \\
\text { increase by } 1-2^{\circ} \mathrm{C} \text { in } \\
\text { winter, Hainan } \\
\text { Province will be } \\
\text { suitable for dengue } \\
\text { transmission all the } \\
\text { year around in future } \\
\text { due to prolonged } \\
\text { infectious life of } \\
\text { mosquito. }\end{array}$ & $\begin{array}{l}\text {-Only considered } \\
\text { the role of } \\
\text { temperature } \\
\text {-No statistical } \\
\text { methods }\end{array}$ \\
\hline $\begin{array}{l}\text { Zheng et al. } \\
\text { (2001) Chinese } \\
\text { [61] }\end{array}$ & $\begin{array}{l}\text { Fuzhou City, } \\
\text { Fujian Province } \\
\text { (2000-2001) }\end{array}$ & $\begin{array}{l}\text { Monthly mean } \\
\text { temperature, relative } \\
\text { humidity, rainfall }\end{array}$ & $\begin{array}{l}\text { Larva Density, } \\
\text { House Index, } \\
\text { Container Index, } \\
\text { Breteau index, } \\
\text { case counts }\end{array}$ & $\begin{array}{l}\text {-Descriptive } \\
\text { analysis }\end{array}$ & $\begin{array}{l}\text {-The temperature and } \\
\text { rainfall played a } \\
\text { considerable role in } \\
\text { vector density and } \\
\text { dengue transmission, } \\
\text { whereas relative } \\
\text { humidity showed a } \\
\text { little relationship. }\end{array}$ & $\begin{array}{l}\text {-Various mosquito } \\
\text { density index } \\
\text { used. Study } \\
\text { period is relative } \\
\text { short }\end{array}$ \\
\hline
\end{tabular}

incidence in the cool months in the rain forest area of Mengla County, Yunnan province, indicating increased risk of transmission as a result of warmer winters [27]. Although global warning could make more areas climatologically suitable for malarial transmission; because higher temperature promotes mosquito development, virus replication and feeding frequency of mosquitoes, extreme high temperature can also restrict the growth of mosquitoes and reduce the spread of malaria. Typically, temperatures lower than $16^{\circ} \mathrm{C}$ or higher than $30^{\circ} \mathrm{C}$ have a negative impact on the development and activities of mosquitoes [30].

High relative humidity is expected to prolong the life of the mosquito enabling it to transmit the infection to several persons. Correlations between relative humidity and malarial transmission were also detected in some regions in China $[19,20,23-25,30,32,39,40,46]$. According to results from regression models based on 15 years of data, Yang et al. (2010) found that relative humidity was more important than rainfall and temperature in addressing the climate-malaria relationship in China [23]. In Motou County of Tibet, Huang et al. (2011) found that relative humidity, which was greatest relative to malaria incidence among meteorological variables as it is a result of temperature, rainfall and other climatic indicators and influenced the activity of mosquito directly such as biting rate and breeding rate [20]. The distribution of mosquitoes, which also is also dependent on relative humidity, determines the extent of malarial spread. Thus, no malaria transmission occurs where the monthly average relative humidity is lower than $60 \%$ [23]. Conversely, it was reported that relative humidity is not a restricting factor in areas where it is higher than 60\%, but temperature then becomes the major driver [23]. For example, no association of relative humidity and malaria transmission was detected in Hainan province $[24,42]$ and the tropical rain forest regions of Yunnan provinces [27], where the relative humidity throughout the years is much higher than $60 \%$. This indicates that it 
Table 4 Characteristics of studies on the association between climatic variables and JE transmission

\begin{tabular}{|c|c|c|c|c|c|c|}
\hline \multirow{2}{*}{$\begin{array}{l}\text { Study \& } \\
\text { Language }\end{array}$} & \multirow{2}{*}{$\begin{array}{l}\text { Study area \& } \\
\text { period }\end{array}$} & \multicolumn{2}{|l|}{ Data Collection } & \multirow{2}{*}{$\begin{array}{l}\text { Statistical } \\
\text { Methods }\end{array}$} & \multirow[t]{2}{*}{ Main findings } & \multirow[t]{2}{*}{ Comments } \\
\hline & & Risk factors & Disease/vector & & & \\
\hline \multirow{2}{*}{$\begin{array}{l}\text { Lin et al. } \\
(2010) \text { English } \\
{[62]}\end{array}$} & \multirow{2}{*}{$\begin{array}{l}\text { Linyi city, } \\
\text { Shangdong } \\
\text { Province 1956- } \\
2004\end{array}$} & \multirow{2}{*}{$\begin{array}{l}\text { Monthly average } \\
\text { temperature, relative } \\
\text { humidity, total rainfall. } \\
\text { Vaccination }\end{array}$} & \multirow[t]{2}{*}{ Monthly incidence } & -Cross-correlation & \multirow[b]{2}{*}{$\begin{array}{l}\text {-Monthly average } \\
\text { temperature and } \\
\text { relative humidity with } \\
\text { no lag were positively } \\
\text { associated with the JE } \\
\text { incidence after } \\
\text { adjusting for the } \\
\text { effect of vaccination. }\end{array}$} & \multirow{2}{*}{$\begin{array}{l}\text {-Vaccination effect } \\
\text { was adjusted, but } \\
\text { only treated as a } \\
\text { simple binary variable. }\end{array}$} \\
\hline & & & & -ARIMA model & & \\
\hline \multirow[t]{4}{*}{$\begin{array}{l}\text { Bi et al. (2007) } \\
\text { English [63] }\end{array}$} & \multirow{4}{*}{$\begin{array}{l}\text { Jinan city, } \\
\text { Shangdong } \\
\text { Province 1959- } \\
1979\end{array}$} & \multirow{4}{*}{$\begin{array}{l}\text { Monthly mean } \\
\text { maximum } \\
\text { temperature, } \\
\text { minimum } \\
\text { temperature, relative } \\
\text { humidity, rainfall and } \\
\text { air pressure. }\end{array}$} & \multirow[t]{4}{*}{ Case counts } & $\begin{array}{l}\text {-Spearman } \\
\text { correlation }\end{array}$ & \multirow{3}{*}{$\begin{array}{l}\text {-The JE incidence was } \\
\text { positively associated } \\
\text { with two temperature } \\
\text { variables, rainfall and } \\
\text { relative humidity, and } \\
\text { negatively correlated } \\
\text { with air pressure. Lag } \\
\text { times were from one } \\
\text { to two months }\end{array}$} & \multirow{3}{*}{$\begin{array}{l}\text {-A potential threshold } \\
\text { of the effect of } \\
\text { temperature was } \\
\text { detected. } \\
\text {-The effect of the } \\
\text { vaccination was very } \\
\text { limited during the } \\
\text { study period of this } \\
\text { study. }\end{array}$} \\
\hline & & & & $\begin{array}{l}\text {-Poisson } \\
\text { regression }\end{array}$ & & \\
\hline & & & & \multirow[t]{2}{*}{$\begin{array}{l}\text {-The Hockey Stick } \\
\text { model }\end{array}$} & & \\
\hline & & & & & $\begin{array}{l}\text {-Thresholds of } 25.2^{\circ} \mathrm{C} \\
\text { for maximum } \\
\text { temperature and } \\
21.0^{\circ} \mathrm{C} \text { were } \\
\text { indentified. }\end{array}$ & $\begin{array}{l}\text {-Non-climatic factors } \\
\text { were neglected }\end{array}$ \\
\hline \multirow[t]{2}{*}{$\begin{array}{l}\text { HSU et al. } \\
\text { (2008) English } \\
{[64]}\end{array}$} & \multirow[t]{2}{*}{ Taiwan 1991-2005 } & \multirow[t]{2}{*}{$\begin{array}{l}\text { Monthly temperature } \\
\text { and precipitation Pig } \\
\text { density Vaccination }\end{array}$} & \multirow[t]{2}{*}{ Case counts } & \multirow[t]{2}{*}{$\begin{array}{l}\text {-Poisson } \\
\text { regression }\end{array}$} & $\begin{array}{l}\text {-The monthly } \\
\text { temperature and } \\
\text { precipitation with two } \\
\text { months lags and the } \\
\text { pig density were } \\
\text { significantly } \\
\text { associated with JE } \\
\text { cases. }\end{array}$ & \multirow[t]{2}{*}{$\begin{array}{l}\text {-Adjustment for } \\
\text { vaccination, pig } \\
\text { density and seasonal } \\
\text { factors. }\end{array}$} \\
\hline & & & & & $\begin{array}{l}\text {-No significant } \\
\text { relationship between } \\
\text { vaccination rate and } \\
\text { counts of JE cases } \\
\text { was found. }\end{array}$ & \\
\hline \multirow[t]{2}{*}{$\begin{array}{l}\text { Bi et al. (2003) } \\
\text { English [65] }\end{array}$} & \multirow{2}{*}{$\begin{array}{l}\text { Jieshou County, } \\
\text { Anhui Province } \\
\text { 1980-1996 }\end{array}$} & \multirow{2}{*}{$\begin{array}{l}\text { Monthly mean } \\
\text { maximum } \\
\text { temperature, } \\
\text { minimum } \\
\text { temperature and } \\
\text { rainfall }\end{array}$} & \multirow[t]{2}{*}{ Monthly incidence } & $\begin{array}{l}\text {-Spearman } \\
\text { correlation }\end{array}$ & \multirow{2}{*}{$\begin{array}{l}\text {-The monthly } \\
\text { minimum } \\
\text { temperature and } \\
\text { precipitation had a } \\
\text { significant relationship } \\
\text { with JE incidence, } \\
\text { with a one-month lag }\end{array}$} & \multirow{2}{*}{$\begin{array}{l}\text {-Vaccination and } \\
\text { other non-climatic } \\
\text { factors were } \\
\text { neglected }\end{array}$} \\
\hline & & & & $\begin{array}{l}\text {-Multiple linear } \\
\text { regression }\end{array}$ & & \\
\hline \multirow{2}{*}{$\begin{array}{l}\text { Huo et al. } \\
\text { (2011) Chinese } \\
{[66]}\end{array}$} & \multirow{2}{*}{$\begin{array}{l}\text { Hebei Province } \\
\text { Tianjin City Beijing } \\
\text { City Inner } \\
\text { Mongolia Shanxi } \\
\text { Province 1994- } \\
2000\end{array}$} & \multirow{2}{*}{$\begin{array}{l}\text { Annual mean } \\
\text { temperature, } \\
\text { maximum } \\
\text { temperature, relative } \\
\text { humidity, minimum } \\
\text { humidity, rainfall and } \\
\text { duration of sunshine }\end{array}$} & \multirow[t]{2}{*}{ Annual incidence } & \multirow[t]{2}{*}{$\begin{array}{l}\text {-Poisson } \\
\text { regression }\end{array}$} & \multirow{2}{*}{$\begin{array}{l}\text {-The annual incidence } \\
\text { of JE was found to be } \\
\text { positively correlated } \\
\text { with annual mean } \\
\text { relative humidity and } \\
\text { negatively associated } \\
\text { with duration of } \\
\text { sunshine }\end{array}$} & $\begin{array}{l}\text {-Yearly variables were } \\
\text { use }\end{array}$ \\
\hline & & & & & & $\begin{array}{l}\text {-Non-climatic factors } \\
\text { were neglected }\end{array}$ \\
\hline $\begin{array}{l}\text { Xu et al. (2009) } \\
\text { Chinese [67] }\end{array}$ & $\begin{array}{l}\text { Tongren area, } \\
\text { Guizhou Province } \\
\text { 1983-2003 }\end{array}$ & $\begin{array}{l}\text { Monthly mean } \\
\text { temperature, air } \\
\text { pressure, relative }\end{array}$ & Case counts & $\begin{array}{l}\text {-Multiple } \\
\text { regression } \\
\text { analysis. }\end{array}$ & $\begin{array}{l}\text {-Among various } \\
\text { climatic variables, the } \\
\text { transmission of JE was }\end{array}$ & $\begin{array}{l}\text {-Non-climatic factors } \\
\text { such as vaccination } \\
\text { were not adjusted }\end{array}$ \\
\hline & & $\begin{array}{l}\text { humidity, rainfall, } \\
\text { wind velocity, } \\
\text { duration of sunshine }\end{array}$ & & & $\begin{array}{l}\text { only correlated with } \\
\text { duration of sunshine. }\end{array}$ & $\begin{array}{l}\text {-Only one area were } \\
\text { analyzed }\end{array}$ \\
\hline $\begin{array}{l}\text { Gao et al. } \\
\text { (2009) Chinese }\end{array}$ & $\begin{array}{l}\text { Guiyang City, } \\
\text { Guizhou Province }\end{array}$ & $\begin{array}{l}\text { Annual mean } \\
\text { temperature and }\end{array}$ & Annual incidence & $\begin{array}{l}\text {-Descriptive } \\
\text { analysis }\end{array}$ & $\begin{array}{l}\text {-Temperature and } \\
\text { precipitation were }\end{array}$ & $\begin{array}{l}\text {-Fifty years long-term } \\
\text { data were collected }\end{array}$ \\
\hline & 1956-2005 & $\begin{array}{l}\text { precipitation Monthly } \\
\text { mean temperature }\end{array}$ & & & $\begin{array}{l}\text { correlated with the } \\
\text { incidence of JE, } \\
\text { especially in July. }\end{array}$ & $\begin{array}{l}\text {-Non-climatic factors } \\
\text { such as vaccination } \\
\text { were ignored. }\end{array}$ \\
\hline
\end{tabular}


Table 4 Characteristics of studies on the association between climatic variables and JE transmission (Continued)

\begin{tabular}{|c|c|c|c|c|c|c|}
\hline & & $\begin{array}{l}\text { and precipitation of } \\
\text { June, July and August. }\end{array}$ & & & & \\
\hline $\begin{array}{l}\text { Liu et al. } \\
\text { (2008) Chinese } \\
{[69]}\end{array}$ & $\begin{array}{l}\text { Kaijiang County, } \\
\text { Sichuan Province } \\
1975-1993\end{array}$ & $\begin{array}{l}\text { Mean temperature, } \\
\text { relative humidity, } \\
\text { rainfall, duration of } \\
\text { sunshine during } \\
\text { November and } \\
\text { December, July and } \\
\text { August, January and } \\
\text { June respectively. }\end{array}$ & Annual incidence & $\begin{array}{l}\text {-Correlation } \\
\text { analysis } \\
\text {-Grey correlation } \\
\text { analysis }\end{array}$ & $\begin{array}{l}\text {-Duration of sunshine } \\
\text { and temperature } \\
\text { were most closely } \\
\text { associated with JE } \\
\text { incidence. }\end{array}$ & $\begin{array}{l}\text {-Only one county was } \\
\text { analyzed } \\
\text {-Annual indicators } \\
\text { were used } \\
\text {-Non-climatic factors } \\
\text { such as vaccination } \\
\text { were ignored. }\end{array}$ \\
\hline $\begin{array}{l}\text { Qu et al. } \\
\text { (2006) Chinese } \\
{[70]}\end{array}$ & $\begin{array}{l}\text { Chaoyang City, } \\
\text { Liaoning Province } \\
\text { 1981-1994 }\end{array}$ & $\begin{array}{l}\text { Annual mean air } \\
\text { pressure, precipitation, } \\
\text { air temperature, } \\
\text { ground temperature, } \\
\text { maximum air } \\
\text { temperature, } \\
\text { minimum ground } \\
\text { temperature, } \\
\text { evaporation and } \\
\text { extreme maximum } \\
\text { and minimum } \\
\text { temperature }\end{array}$ & Annual incidence & $\begin{array}{l}\text {-Correlation } \\
\text { analysis } \\
\text {-Back propagation } \\
\text { artificial neural } \\
\text { network }\end{array}$ & $\begin{array}{l}\text {-The JE incidence was } \\
\text { negatively correlated } \\
\text { with air pressure, and } \\
\text { positively correlated } \\
\text { with evaporation, } \\
\text { maximum } \\
\text { temperature and } \\
\text { extreme maximum } \\
\text { temperature. }\end{array}$ & $\begin{array}{l}\text {-Various } \\
\text { meteorological factors } \\
\text { were applied } \\
\text {-The predictive ability } \\
\text { of the BP neural } \\
\text { network model is not } \\
\text { very strong. }\end{array}$ \\
\hline $\begin{array}{l}\text { Zhang et al. } \\
\text { (2004) Chinese } \\
{[71]}\end{array}$ & $\begin{array}{l}\text { Dali, Yunnan } \\
\text { Province 1992- } \\
2001\end{array}$ & $\begin{array}{l}\text { Mean temperature in } \\
\text { May, rainfall in } \\
\text { September, annual } \\
\text { mean temperature, } \\
\text { rainfall estimated } \\
\text { vaccination coverage, } \\
\text { paddy field areas }\end{array}$ & Annual incidence & $\begin{array}{l}\text {-Correlation } \\
\text { analysis } \\
\text {-Multiple } \\
\text { regression }\end{array}$ & $\begin{array}{l}\text {-The annual JE } \\
\text { incidence was found } \\
\text { to be correlated with } \\
\text { temperature and } \\
\text { rainfall. No } \\
\text { relationships between } \\
\text { the JE incidence and } \\
\text { estimated vaccination, } \\
\text { as well as paddy field } \\
\text { areas were found. }\end{array}$ & $\begin{array}{l}\text {-Use of approximate } \\
\text { estimated vaccination } \\
\text { data } \\
\text {-Data of paddy field } \\
\text { areas were collected. }\end{array}$ \\
\hline $\begin{array}{l}\text { Liu et al. } \\
\text { (2003) Chinese } \\
{[72]}\end{array}$ & $\begin{array}{l}\text { Chaoyang City, } \\
\text { Liaoning Province } \\
\text { 1983-2002 }\end{array}$ & $\begin{array}{l}\text { Mean temperature } \\
\text { and rainfall during } \\
\text { June and August, } \\
\text { annual mean rainfall }\end{array}$ & Annual incidence & $\begin{array}{l}\text {-Correlation } \\
\text { analysis } \\
\text {-Multiple } \\
\text { regression }\end{array}$ & $\begin{array}{l}\text {-The annual JE } \\
\text { incidence was just } \\
\text { correlated to the } \\
\text { rainfall in July among } \\
\text { climatic factors } \\
\text { selected. }\end{array}$ & $\begin{array}{l}\text {-Non-climatic factors } \\
\text { such as vaccination } \\
\text { were ignored. } \\
\text {-Annual incidence } \\
\text { was used }\end{array}$ \\
\hline $\begin{array}{l}\text { Shen et al. } \\
\text { (2002) Chinese } \\
{[73]}\end{array}$ & $\begin{array}{l}\text { Shanghai 1952- } \\
1997\end{array}$ & $\begin{array}{l}\text { Monthly temperature } \\
\text { of June, July and } \\
\text { August respectively, } \\
\text { total rainfall of June } \\
\text { and July Areas of rice } \\
\text { field, pig rising, } \\
\text { mosquito density, } \\
\text { vaccination rate }\end{array}$ & Annual incidence & $\begin{array}{l}\text {-Descriptive } \\
\text { analysis }\end{array}$ & $\begin{array}{l}\text {-No obvious } \\
\text { relationships between } \\
\text { JE incidence and } \\
\text { climatic factors and } \\
\text { areas of rice field as } \\
\text { well as pig rising } \\
\text { were observed, } \\
\text { implying that the } \\
\text { decrease of JE } \\
\text { incidence during } \\
\text { study period may be } \\
\text { due to massive } \\
\text { vaccination } \\
\text { conducted in } \\
\text { Shanghai. }\end{array}$ & $\begin{array}{l}\text {-Both climatic and } \\
\text { non-climatic data } \\
\text { were collected } \\
\text {-Climatic variables } \\
\text { only in three months } \\
\text { were analysis }\end{array}$ \\
\hline $\begin{array}{l}\text { Zhang et al. } \\
\text { (1997) Chinese } \\
{[74]}\end{array}$ & $\begin{array}{l}\text { Henan Province } \\
\text { Not specific }\end{array}$ & $\begin{array}{l}\text { Temperature, rainfall } \\
\text { Elevation }\end{array}$ & $\begin{array}{l}\text { Case count JE } \\
\text { incidence }\end{array}$ & $\begin{array}{l}\text {-Correlation } \\
\text { analysis }\end{array}$ & $\begin{array}{l}\text {-The JE incidence was } \\
\text { positively correlated } \\
\text { with temperature and } \\
\text { rainfall, but decreased } \\
\text { with increased } \\
\text { elevation. }\end{array}$ & $\begin{array}{l}\text {-The impact of } \\
\text { vaccination was } \\
\text { ignored } \\
\text {-Data collection was } \\
\text { not described clearly }\end{array}$ \\
\hline $\begin{array}{l}\text { Feng et al. } \\
\text { (1996) Chinese } \\
{[75]}\end{array}$ & $\begin{array}{l}\text { Fengyi of Eyuan } \\
\text { County, Dali, } \\
\text { Yunnan Province } \\
1991\end{array}$ & $\begin{array}{l}\text { Monthly mean } \\
\text { temperature and } \\
\text { rainfall }\end{array}$ & Monthly incidence & $\begin{array}{l}\text {-Descriptive } \\
\text { analysis }\end{array}$ & $\begin{array}{l}\text {-The monthly } \\
\text { incidence was found } \\
\text { to be related to } \\
\text { monthly temperature } \\
\text { and rainfall }\end{array}$ & $\begin{array}{l}\text {-Only one year data } \\
\text { was analysed }\end{array}$ \\
\hline
\end{tabular}


is not necessary to consider humidity when making malaria epidemic predictions in areas of consistently high humidity.

The impact of precipitation on malaria transmission is inconsistent across geographical locations in China. Some studies indicated that rainfall was closely correlated to malaria incidence [19-21,23-26,30-35,37,39,40], whereas some failed to detect such an association $[22,27,36]$. Rainfall not only provides the medium for the mosquito life cycle, but is also related to high humidity, thereby enhancing mosquito survival. Using Bayesian hierarchical models, Huang et al. (2011) showed that the way rainfall influenced malaria incidence in central China was different from other climatic factors. This implies that malaria incidence is more sensitive to rainfall compared to other meteorlogic variables [20]. However, the association between mosquito abundance and rainfall is non-linear. Excessive rainfall often leads to small puddles serving as mosquito breeding sites and therefore increases malaria transmission. But heavy rain may destroy existing breeding places and flush the eggs or larvae out leading to reduced transmission [25,30]. A negative effect of rainfall on malaria spread was detected by Wang et al. in Anhui province, which identified that every $1 \mathrm{~mm}$ annual rainfall increase corresponds to $27 \%$ decrease of malaria cases [35]. Fog precipitation, as another important source of water in many mountainous and coastal regions, was first found to be a predictor of malaria in the tropical rain forest area of Mengla County, southwest China by Tian et al. [27].

In some studies ecological proxy indicators such as Normalized Difference Vegetation Index (NDVI) [29,35, $37,39,43]$ and South Oscillation Index (SOI) [28] were also analyzed for the detection of the climate-malaria relationship. For example, in southeastern Yunnan Province, remote sensing NDVI was found to be a sensitive evaluation index of Anopheles density and malaria incidence rate by using grey correlation analysis [29]. The EI Nino-Southern Oscillation (ENSO) represents a periodic variation in the atmospheric conditions and ocean surface temperatures of the tropical Pacific and was determined to have a positive influence on malaria incidence in Anhui province, China [28].

The effect of climatic factors on mosquito-borne diseases including malaria is not immediate and usually results in a lag-time due to the life cycle of the vector and the parasite [20]. Lag times of different climate indicators were analyzed in some studies on different geographical and temporal scales. For example, Zhou et al. (2010) reported a $75.3 \%$ change in monthly malaria incidence was correlated with the average monthly temperature, the average temperature of last two months and the average rainfall of current month in central China [21]. In Yunnan Province, obvious associations between both $P$. vivax and P. falciparum malaria and climatic factors with a clear one-month lagged effect were found [25]. It is essential to take lag effect into account in addressing the impact of climate change at a local level because it provides important information for early detection and warning for mosquito-borne diseases.

\section{Dengue fever}

Dengue fever is the most common arboviral disease in the tropics and subtropics, and about 2.5 billion people live in regions at risk for dengue transmission [77]. Since the first recorded outbreak of dengue fever in Foshang City in 1978, dengue occurs frequently in southern China, including Guangdong, Guangxi, Hainan, Taiwan, Fujian, Zhejiang and Yunnan [78]. In China, Aedes albopictus is the most important mosquito in dengue transmission in China. Due to its wider geographic distribution it could be responsible for recent dengue outbreaks in Guangzhou and Zhejiang Province [79]. As another major vector of dengue virus, distribution of Aedes aegypti which was previously considered only in the coastal areas of the tropical zone below $22^{\circ} \mathrm{N}$ latitude has already extended into regions of $25^{\circ} \mathrm{N}$ latitude, such as Yunnan Province [80].

Recently, the impacts of climate change on dengue transmission and dengue vector distribution in China have been evaluated and identified in limited studies [47-61]. Although meteorology alone does not initiate dengue epidemics and it is reported that there appears to be a smaller climatic effect on this disease than occurs with other arboviruses [81]. Temperature, rainfall and relative humidity were considered major meteorological determinants in most of studies, whereas one study from Cixi, Zhejian Province reported no correlation between dengue outbreaks and climatic factors [53]. This is perhaps due to the non-endemic nature of dengue in Cixi and a short (4-month) study period.

Although results of studies with varied temporal, spatial or spatiotemporal approaches are not consistent in terms of the effect of temperature $[82,83]$, historical data do suggest that temperature plays an important role in vector competence and dengue transmission [84-86]. Some studies in China have also identified an obvious association between temperature variables and vector distribution, dengue outbreaks and distribution. In Hainan Province, Chen et al. (2002) revealed that under global warming conditions, Hainan would be suitable for continuous dengue transmission with dengue fever cases year-round [60]. Similarly, Yu et al. (2005) reported that over half of the area of Hainan would be favorable for year-round dengue transmission by 2030 and 2050 based on predicted winter temperatures [57]. Using the CLIMEX model, Wu et al. found that due to summer expansion, Ae. albopictus have extended their geographic range to 
areas which experienced an annual mean temperature below $11^{\circ} \mathrm{C}$ and a January mean temperature below $-5^{\circ} \mathrm{C}$. This finding highlights that most provinces and cities in China now support survival and development of $A e$. albopictus and risk the occurrence of dengue fever or the establishment of dengue virus in the mosquito population [47]. In subtropical Taiwan, it was found that every $1^{\circ} \mathrm{C}$ increase of monthly average temperature could lead to 1.96 times increase of the total population at risk for dengue fever transmission, indicating that a slight increase in temperature could result in epidemics of this disease [55]. Therefore, climate change, particularly a warming trend, increases the land area suitable for disease vectors,altering or increasing dengue fever distribution. Furthermore, other climatic indicators such as rainfall, relative humidity and wind velocity together with temperature can be significant predictors of dengue incidence. For example, Lu et al. (2009) showed that in Guangzhou City minimum temperature and minimum humidity, at a lag of one month, were positively associated with dengue incidence, whereas an obvious negative effect of wind velocity on dengue cases was observed in the same month [51].

Unfortunately, less work was conducted to relate dengue outbreaks and climatological events in mainland China. Several studies in Taiwan reported that typhoons remain an important factor affecting vector population and dengue fever $[48,52]$. Lai (2011) found that two outbreaks of dengue and increasing vector population in Kaohsiung, Taiwan in 2002 and 2005 were exacerbated by hot and wet climate conditions caused by warm sea surface temperatures and typhoon weather patterns [48]. Typhoons could result in massive rainfall, high humidity and water pooling resulting in mosquito breeding sites [48]. Conversely, a sharp drop in temperature and substantial rainfall caused by frequent typhoons may contribute to a temporary reduction in dengue infections [52]. More research on the impact of extreme climatic conditions such as floods, droughts, typhoons and storms on mosquito-borne diseases are needed in China.

Along with climatic drivers, many site-specific variations in some factors affecting dengue transmission, such as mosquito density, imported cases and other environmental factors were also identified and highlighted in a few studies in China [48-50,53,55,59]. Indices such as the Breteau Index (BI), Housing Index (HI) and Container Index $(\mathrm{CI})$ were traditionally employed to determine mosquito density. In Kaohsiung city, Taiwan, the $\mathrm{BI}$, which indicates the number of positive containers per 100 houses, in addition to local climatic factors were found to be good predictors of dengue incidence [49]. Similarly, Lai et al. (2011) demonstrated that the number of dengue fever admissions in Kaohsiung city was significantly correlated with BI with a time lag of 32 and 22 days during both summer and autumn in 2002 and
2005, respectively [48]. From a spatial standpoint, one study examined the relationships among weather profiles, environmental factors of interest, socioeconomic factors and geographical distributions of dengue fever and showed the number of months with average temperature higher than $18^{\circ} \mathrm{C}$ and the level of urbanization were significantly associated with dengue fever risk at the township level in Taiwan [54]. Using logistic and Poisson regression models, Shang et al. (2010) emphasized the importance of imported case and favorable climate conditions in the initiation of dengue epidemics, also highlighting that the development of an early warning surveillance system, utilizing relevant meteorological information, will be an invaluable tool for prevention and control of dengue fever [50]. Other environmental and host factors, such as intervention measures and human risk behaviors, also influence mosquito populations and the extent of dengue spread. Thus, more work should be conducted in the future for a better understanding of these complex interactions.

\section{Japanese Encephalitis}

Japanese encephalitis (JE), a mosquito-borne viral disease, is mostly transmitted by Culex tritaeniorhynchus in China, with pigs as a reservoir host and source of infection [87]. Due to mass vaccination the 1980s and improved economic circumstances in China, the morbidity and mortality due to JE has declined gradually each year [88]. Over a 6-year period between 2000 and 2005, the annual incidence decreased from $0.9489 / 100,000$ to 0.3898 / 100,000 [88]. Similar findings were also observed in Taiwan. Since mass vaccination was implemented in 1968, the incidence of JE has declined from 2.05/10000 to 0.03/ 10000 in ten years from 1967 to1997 in Taiwan [89]. However, JE is still one of an acute epidemic disease posing a threat to public health, and it has recently spread to new territories [88]. In 2009, JE virus was isolated from $\mathrm{Cu}$. tritaeniorhynchus mosquitoes collected in Tibet, indicating that JE virus has extended its geographical range to a region that was previously non-endemic due to high elevation [90]. Such trends in geographical spread of JE were also recently reported in other countries such as Australia [91-93]. Highly endemic areas of JE in China include Sichuan Province, Guizhou Province, Chongqing Municipality, Yunnan Province and Shaanxi Province, which are mainly located in southwest and central China. The five provinces account for $50 \%$ of the total cases nationwide [88].

Global warming might change temperature and rainfall patterns [94,95], which may affect the development and infection capacity of both the mosquito and the virus. Relative humidity is also important in the transmission of JE because mosquitoes can survive longer and disperse further in areas with suitable relative 
humidity [96]. Studies in different areas of Asia have also shown the likely influence of climate on the incidence of JE [97-99]. However, little research has been conducted to examine the effect of climatic variables, along with mass vaccination and other non-climatic drivers in China. Bi et al. (2007) have identified positive relationships between climatic variables (monthly maximum temperature, minimum temperature and total rainfall) and JE transmission in a rural region of Anhui Province [65] and a metropolitan area of Shangdong Province [63] where no rice was grown and the role of pigs in disease transmission was not fully understood [63]. Unfortunately, the effects of vaccination on JE control in the two areas were very limited during the study periods. In the metropolitan area of Jinan city, a potential threshold of the effect of temperature on JE was also detected by the Hockey Stick model which is based on the assumption that temperature has no effect on JE cases until a threshold value. When the monthly mean maximum temperature was higher $25.2^{\circ} \mathrm{C}$ or the minimum temperature was over $21.0^{\circ} \mathrm{C}$, an obvious increase in JE cases occurred [63]. These findings are consistent with the threshold temperature detection in previous Chinese studies [87]. Using ARIMA models, Lin et al. (2011) suggested that monthly average temperature and relative humidity at 0 -month lag were positively associated with JE incidence in Linyi, another city of Shangdong Province after adjusting for mass vaccination in this area [62]. Time lag-0 of climate variables was perhaps because the behaviour of pig breeding in Linyi, along with the high density of mosquitoes help to shorten the transmission cycle [62]. With adjustment of more interactional factors including seasonal pattern, time trend, pig density, 23 geographic areas representing location of farm and paddy cultivation, and vaccination coverage, HSU et al. (2008) identified the significant effects of monthly temperature and rainfall with two months lag on the monthly occurrence of JE in Taiwan [64]. Similarly, temperature and rainfall were two significant determinants of JE spread with control of vaccination coverage and paddy field areas [71].

Few analyses, without controlling for non-climatic factors that potentially affect JE transmission, also reported associations between different climatic variables such as temperature, rainfall and JE annual incidence by correlation and regression analyses [66-69,72,74]. Using multiple stepwise regression, $\mathrm{Xu}$ et al. (2009) found that among various meteorological indicators the transmission of JE was only correlated with duration of sunshine in Tongren area, Guizhou Province [67]. Similarly, a close relationship between sunshine and annual incidence of JE was also reported by Huo et al. [66] and Liu et al. [69] in north China and Kaijiang county of Sichuan Province in Southwestern China, respectively. Applying correlation analysis and back propagation artificial neural work, the annual JE incidence was found to be negatively correlated with mean air pressure, and positively correlated with mean evaporation, maximum temperature and extreme maximum temperature [70]. In future research investigating the influence of climate change on JE transmission, important factors should be measured and controlled for, such as social- economic status, population immunity (including vaccination), mosquito control measures, pig rising, areas of rice field and the virulence of the virus. Moreover, research is also needed in sporadic- and meso- endemic areas, such as Jiangxi Province, Hunan Province, Fujian Province and Guangdong Province.

\section{Implications for adaptation in China}

The potential effects of climate change on the spatial and temporal distribution of mosquito-borne diseases and vectors in China have been summarized in this review. Furthermore, the synthesis of the literature shows an urgent need for improving current control policies and developing targeted adaptive strategies in China to address mosquitoborne disease. Although some health benefits have also been achieved through mitigation policies in China, adaptation, preparing to manage some of the unavoidable effects of climate change on human health, is another important response strategy [100]. Based in part on recommendations and established approaches in recent studies of adaptation to climate change [81,101-105], as well as on the status of mosquito-borne diseases in China, we highlight five principles to guide timely development of adaptation mechanism to reduce the adverse impacts of climate change on the control of mosquito-borne diseases. These guidelines may also be applicable in addressing the threat to other health outcomes from climate change.

\section{Improving current surveillance and monitoring systems integrated with climate-sensitive conditions}

In recent years, the Chinese government has paid great attention to the prevention and control of mosquitoborne diseases. Since 2004, cases of infectious diseases have been electronically recorded and the data collected at the national level by the Chinese Center for Diseases Control and Prevention (China CDC). This important step means the disease surveillance system is more sensitive and efficient than in previous years. However, unavailability of good quality long-term data sets has hampered our understanding of the likely impacts of climate change on mosquito-borne diseases and vector distribution. Kovats et al. (2001) have pointed out that climate change and health researches require at least 30 years of data because short- and medium- term associations may not provide an accurate picture of the impact of climate change occurring over many decades [106]. In China, imperfect data collected by passive surveillance systems restrict such climate-health relationship analysis. 
For example, onset dates rather than notification dates are not available, which may lead to considerable information bias. According to a national report in 2005, it was also estimated that only $1 / 18(5.6 \%)$ malaria cases in China are reported [107]. Furthermore, lack of routine data collection of vectorial indicators has restricted our understanding of real geographic and temporal distributions of mosquito vectors.

Under the circumstance of the changing climate, truly effective surveillance systems and monitoring systems can be used to identify changes in the range and incidence of diseases; determine whether these changes are to be the result of climate change; assist the development of response measures and develop hypotheses about the climate-health relationship [108]. We believe that there are also many gaps for improving current surveillance and monitoring of infectious diseases in China in response to climate variation. Surveillance for early detection of epidemics of mosquito-borne diseases based on readily climatic data, such as daily temperature records, in combination with other interactional factors are of paramount importance [108]. For example, an effective early warning system for outbreaks of mosquitoborne diseases based on predicted extreme weather conditions, such as extreme temperature or rainfall, can be considered as an immediate opportunity for adaptation by strengthening the preparedness of emergency response before periods of high-risk [100]. Careful tracking of imported cases, in conjunction with relevant meteorological data, is also of assistance in providing earlier warning signals for emerging indigenous epidemics [109,110]. In short, coherent surveillance systems integrated with climate-sensitive conditions are urgently needed to improve scientific knowledge about the health risks of climate change, and to prioritize needs for intervention and adaptation options.

\section{Focusing adaptation strategies and policies on vulnerable communities}

Some populations and geographical regions will be particularly vulnerable to climate change. Although climate change is a global threat to public health, it is well acknowledged that poorer nations and communities who have contributed least to greenhouse gas emissions are most vulnerable to the effects [111]. Higher malaria risk in China has also been associated with poverty, poor quality housing, unhygienic surroundings and agricultural activities in rural and remote areas. Chinese farmers in rural regions who usually work in fields and sleep in the open are at higher risk of mosquito biting especially in summer and autumn, when the peak times of malaria occurred due to favorable climate conditions and active propagation of mosquitoes. However, urban populations may also share some increased vulnerabilities in the context of climate change. For example, populations living in cities located on the coast or on small islands may be particularly vulnerable to frequent rainfall and storms and are also exposed to changes in the spread of mosquitoborne diseases, such as Taiwan. Additionally, metropolitan regions may be experiencing two types of warming trends; warmer temperatures caused by the urban heat island effect as well as global climate change, which could make more urban areas suitable for the transmission of mosquito-borne diseases by reducing development times, increasing survival probabilities and biting frequency for the mosquitoes [102,112].

Due to limited funding, resources, and time, effective adaptive action is required to protect the most vulnerable individuals and communities, due to geographic locations and low adaptive capacity, from inevitable effects of climate change on mosquito-borne diseases. Firstly, better identification of real vulnerable groups needs to be based on more comprehensive factors, such as political rigidity, population growth, poverty, culture, dependency, geographic isolation, population immunity and human perceptions, behaviours and activities etc. This requires both qualitative and quantitative assessing methods in future adaptation research. The next step is the development and implementation of timely and efficient adaptive strategies in those targeted communities by collective coordination of all relevant sectors. Adaptive options may include expanding mosquito control, improving vaccination coverage, enhancing existing elimination programs and conducting health education programs in a relative short term, and the establishing an early warning system, improving housing quality, strengthening preparedness and response of extreme weather events (e.g. better and adequate urban drainage systems) in a long run.

\section{Strengthening the capacity of public health system to adapt climate change}

In the Chinese context, an efficient network system of mosquito-borne diseases control has been established and CDCs at national, province, and city level take the major responsibility in the network. Although the public health system has credible skills and experience ranging in disease control and prevention, public health management and emergency preparedness services, the status of the resources and capacity of mosquito-borne diseases control at local levels is not optimistic, especially in rural counties, townships and communities. For example, Chen et al. (2010) pointed out that lack of additional funding, additional staff, staff straining and equipment currently has become the major hamper of improving ability of local public health sectors in malaria surveillance, diagnosis and treatment, and mosquito control [113]. 
The climate change will have local impacts, and a significant share of dealing with the adverse impacts of the change will fall on local public health arena. Making climate change adaptation a priority for local public health sectors, however, is challenged in current China due to a chronic lack of resources and limited awareness and knowledge about health impacts of climate change. Disappointedly, health implications of climate change have largely neglected in not only developing countries but developed ones, compared with its energy, economic and environmental implications. For example, shortage of public health professionals and small part of research funding of climate change for public health were reported among public health department directors in the U.S., which are also a major challenge for China in adapting the changing climate [114]. Moreover, inaccessible information and training on health influences of climate change may restrict awareness, knowledge, attitude and decisions of local public health departments. In brief, adaptation to climate change will require the public health system has a key leadership role to take in health adaptation strategy making and implementation in China. Stable funding, additional staff and better information access will be needed to best prepare the public health sectors to manage the health risks associated with climate change.

\section{Developing multidisciplinary approaches sustained by a new mechanism of intersectoral coordination and collaboration}

What makes addressing the range and magnitude of health impacts of climate change even more difficult is unavoidable complexities and uncertainties in multifactorial causal webs. We can not deal with climatic variables or health outcomes or any other potential interacting drivers in isolation and need to integrate scientific knowledge from various disciplines to tackle these interactions by developing a better collaborated mechanism across all relevant governmental and non-governmental sectors and institutions which are responsible for the prevention and control of climate-sensitive disease [101,112]. Although the existing national plans and policies about climate change clearly indicate that adaptation to health burdens from climate-sensitive diseases is a multiplesector responsibility, mechanisms of intersectoral decision making and coordination do not yet operated to guarantee free and open exchange of information, adequate compliance and participation, ongoing financial and technological support.

For the control and prevention mosquito-borne diseases under condition of climate change, it is even more urgent to produce multidisciplinary insights from diverse public and private sectors. Although some health risks can be reduced largely by health sector interventions such as surveillance, mosquito control, spraying, vaccination, sanitation activities and health education, many adverse impacts require concerted adaptive options with other relevant sectors such as meteorology, environmental, urban designing and planning, water, agriculture and housing [101]. Unfortunately, health sectors in China which invest greater financial and resources support, are often the only ones responsible for disease prevention and control. Therefore, there is a particular need for a multidisciplinary approach sustained by ongoing intersectoral coordination and collaboration, which not only allow us to have a bettering understanding of the complex climate-health relationship, but will provide integrated and practical adaptive strategies to minimize climate-sensitive disease impairments, and further influence policy-formulation and decision-making $[115,116]$.

\section{Promoting awareness and mobilization of the public and individuals}

An important step in the development and promotion of successful local adaptation options is raising public and professional awareness. Governments, institutions, and organizations play indispensable roles in those adaptive actions but so do the public and individuals if they are receptive to behavior change to adapt to a world altered by climate change [101]. It is well acknowledged that the perceived risk of climate change in the population is the strongest motivator of health behaviour change, that is, it is only when individuals feel vulnerable and threatened to the impacts of climate change that they will take autonomous adaptation seriously [117]. In China, climate change has traditionally been treated as an environmental threat rather than a public health issue. We highlight that awareness programs about the health aspects of climate change are urgently needed, coupled with highquality baseline investigations to examine public perception of adverse health effects from climate change in China.

Mobilization of the public to adapt to climate change also depends on availability of information about effective adaptation measures as well as social capacity to deal with these problems. A recent cross-sectional survey conducted in the U.S. showed that people who report knowledge of the necessary information to prepare for adverse health impacts of climate change were more likely to have an adaptive plan for their household [118]. For the prevention of mosquito-borne diseases, scientific knowledge and information, such as risk behaviour and self-protection measures, should be rapidly dispersed during high-risk periods to strengthen the adaptive capacity of the public. For example, relevant health intervention campaigns can be conducted to warn and educate local communities to change personal behaviour, e.g. use of mosquito nets in the field at peak time of 
mosquito-borne diseases; cleaning living conditions as soon as an increase in cases; emptying artificial containers with stagnant water timely. Moreover, better implementation of planned adaptation to climate change requires good social capability which depends on resource availability and cultural acceptability, indicating that successful adaptation strategies much be suitable for local conditions and accepted by local populations [101].

\section{Conclusions}

This study included scientific evidence of impacts of climate change on the transmission of mosquito-borne diseases, resulting in increase in incidence and geographic spread in China. Variability in temperature, precipitation, wind and extreme weather events has been observed to be linked with changes of spatial and temporal distribution of malaria, dengue fever, Japanese encephalitis in some regions in China. However, research to date has limitations and challenges in attributing changes in the status of mosquito-borne diseases to climate change. Potential adverse effects heighten the urgent need to conduct more high-quality research for assessing risks of these climate-sensitive vector-borne diseases, to improve current control policies from a weather-based direction, and to develop targeted policies for adapting short-term and long-term climate shifts in China.

Based on summarization of what is known about the likely impacts of climate change on these diseases in China we highlight five principles to guide policy formulation to enhance adaptation mechanism to reduce the adverse impacts of climate change on the control of mosquito-borne diseases. These recommendations include: 1) improving current surveillance and monitoring systems integrated with climate-sensitive conditions; 2) focusing adaptation strategies and policies on vulnerable communities; 3 ) strengthening public health system capacity to adapt to climate change; 4) developing multidisciplinary approaches sustained by a new mechanism of intersectoral collaboration; and 5) promoting awareness and mobilization of the public as well as health and other professionals.

\section{Abbreviations}

IPCC: Intergovernmental Panel on Climate Change; CHKD: China Hospital Knowledge Database; NDVI: Normalized Difference Vegetation Index; SOI: South Oscillation Index; ARIMA: Autoregressive integrated moving average model; GIS: Geographic Information System; PCA: Principle Component Analysis; P. vivax: Plasmodium vivax; P. falciparum: Plasmodium falciparum; ENSO: El Nino-Southern Oscillation; BI: Breteau Index; HI: Housing Index; Cl: Container Index; JE: Japanese encephalitis; CDC: Center for Diseases Control and Prevention.

\section{Competing interests}

The authors declare that they have no competing interests.

\section{Authors' contributions}

LB and QL designed the study and carried out the literature search. $L B$ and LCM reviewed included studies and wrote the paper. All authors read and approved the final manuscript.

\section{Acknowledgements}

This study was supported by the National Basic Research Program of China (973 Program) (Grant No. 2012CB955504).

\section{Author details}

'State Key Laboratory for Infectious Diseases Prevention and Control, National Institute for Communicable Disease Control and Prevention, Chinese Center for Disease Control and Prevention, Beijing 102206, People's Republic of China. ${ }^{2}$ University of South Florida College of Public Health, 4202 E. Fowler Avenue, Tampa, FL 33620, USA. ${ }^{3}$ Shandong University Climate Change and Health Center, Jinan 250012Shandong, People's Republic China.

Received: 13 January 2012 Accepted: 1 March 2013

Published: 9 March 2013

\section{References}

1. Climate change 2007: Impacts, Adaptation and Vulnerability. In Proceedings of the Working Group II to the Fourth Assessment Report of the IPCC. Edited by Parry ML, Canziani OF, Palutikof JP, van der Linden P, Hanson C. Cambridge: Cambridge University Press; 2007:391-431.

2. McMichael AJ, Githeko AK: Climate change: Impacts, adaptation, and vulnerability. In Human health. Chapter 9. Edited by McCarthy JJ, Canziani OF, Leary NA, Dokken DJ, White KS. Cambridge: Cambridge University Press; 2001.

3. McMichael AJ, Haines A, Slooff R, Kovats S: Climate Change and Human Health. Geneva: World Health Organization (WHO); 1996.

4. Impacts, Adaptations and Mitigation of Climate Change: ScientificTechnical Analyses. In Proceedings of the Working Group II to the Second Assessment of the Intergovernmental Panel on Climate Change (IPCC). Edited by Watson RT, Zinyowera MC, Moss RH. Cambridge: Cambridge University Press; 1996.

5. Watson RT, Zinyowera MC, Moss RH (Eds): The Regional Impacts of Climate Change: An Assessment of Vulnerability. Special Report of the Intergovernmental Panel on Climate Change (IPCC) Working Group II. Cambridge: Cambridge University Press; 1998.

6. Alsop Z: Malaria returns to Kenya's highlands as temperatures rise. Lancet 2007, 370:925-926.

7. Olson SH, Gangnon R, Elguero E, Durieux L, Guegan JF, Foley JA, Patz JA: Links between climate, malaria, and wetlands in the Amazon Basin. Emerg Infect Dis 2009, 15:659-662.

8. Dantur Juri MJ, Zaidenberg M, Claps GL, Santana M, Almiron WR: Malaria transmission in two localities in north-western Argentina. Malar J 2009, 8:18.

9. Pascual M, Ahumada JA, Chaves LF, Rodo X, Bouma M: Malaria resurgence in the East African highlands: temperature trends revisited. Proc Natl Acad Sci 2006, 103:5829-5834.

10. Zhou G, Minakawa N, Githeko AK, Yan G: Association between climate variability and malaria epidemics in the East African highlands. Proc Natl Acad Sci 2004, 101:2375-2380.

11. McMichael AJ, Woodruff RE, Hales S: Climate change and human health: present and future risks. Lancet 2006, 367:859-869.

12. Hay SI, Cox J, Rogers DJ, Randolph SE, Stern DI, Shanks GD, Myers MF, Snow RW: Climate change and the resurgence of malaria in the East African highlands. Nature 2002, 415:905-909.

13. Hay SI, Shanks GD, Stern DI, Snow RW, Randolph SE, Rogers DJ: Climate variability and malaria epidemics in the highlands of East Africa. Trends Parasitol 2005, 21:52-53.

14. Reiter P: Climate change and mosquito-borne disease. Environ Health Perspect 2001, 109(Suppl 1):141-161.

15. Zhou SS, Wang Y, Fang W, Tang LH: Malaria situation in the People's Republic of China in 2007. Zhongguo Ji Sheng Chong Xue Yu Ji Sheng Chong Bing Za Zhi 2008, 26:401-403.

16. Center for Disease Control, D. o. H., Executive Yuan, Taiwan: Statistics of communicable diseases and surveillance report in Taiwan area. Taiwan: Center for Disease Control, Department of Health, Executive Yuan; 2004.

17. Wang LH, Fu SH, Wang HY, Liang XF, Cheng JX, Jing HM, Cai GL, Li XW, Ze WY, Lv XJ, Wang HQ, Zhang DL, Feng Y, Yin ZD, Sun XH, Shui TJ, Li MH, Li YX, Liang GD: Japanese encephalitis outbreak, Yuncheng, China, 2006. Emerg Infect Dis 2007, 13(7):1123-1125.

18. National Development and Reform Commission 2007, National Development and Reform Commission: China's National Climate Change 
Programme. Beijing: National Development and Reform Commission; 2007. Available: http://www.ccchina.gov.cn/WebSite/CCChina/UpFile/File188.pdf.

19. Huang F, Zhou S, Zhang S, Zhang H, Li W: Meteorological factors-based spatio-temporal mapping and predicting malaria in central China. AmJTrop Med Hyg 2011, 85:560-567.

20. Huang F, Zhou S, Zhang S, Wang H, Tang L: Temporal correlation analysis between malaria and meteorological factors in Motuo County, Tibet. Malar Journal 2011, 10:54.

21. Zhou SS, Huang F, Wang JJ, Zhang SS, Su YP, Tang LH: Geographical, meteorological and vectorial factors related to malaria re-emergence in Huang-Huai River of central China. Malar J 2010, 9:337.

22. Zhang Y, Bi P, Hiller JE: Meteorological variables and malaria in a Chinese temperate city: a twenty-year time-series data analysis. Environ Int 2010, 36:439-445.

23. Yang GJ, Gao Q, Zhou SS, Malone JB, McCarroll JC, Tanner M, Vounatsou P, Bergquist $\mathrm{R}$, Utzinger J, Zhou XN: Mapping and predicting malaria transmission in the People's Republic of China, using integrated biologydriven and statistical models. Geospat Health 2010, 5:11-22.

24. Xiao D, Long Y, Wang S, Fang L, Xu D, Wang G, Li L, Cao W, Yan Y: Spatiotemporal distribution of malaria and the association between its epidemic and climate factors in Hainan, China. Malar Journal 2010, 9:185.

25. Hui FM, Xu B, Chen ZW, Cheng X, Liang L, Huang HB, Fang LQ, Yang H, Zhou $\mathrm{HN}$, Yang HL, Zhou XN, Cao WC, Gong P: Spatio-temporal distribution of malaria in Yunnan Province, China. AmJTrop Med Hyg 2009, 81:503-509.

26. Clements AC, Barnett AG, Cheng ZW, Snow RW, Zhou HN: Space-time variation of malaria incidence in Yunnan province, China. Malar Journal 2009, 8:180.

27. Tian L, Bi Y, Ho SC, Liu W, Liang S, Goggins WB, Chan EY, Zhou S, Sung JJ: One-year delayed effect of fog on malaria transmission: a time-series analysis in the rain forest area of Mengla County, south-west China. Malar Journal 2008, 7:110.

28. Bi P, Parton KA, Tong S: El Nino-Southern Oscillation and vector-borne diseases in Anhui, China. Vector Borne Zoonotic Dis 2005, 5:95-100.

29. Liu J, Chen XP: Relationship of remote sensing normalized differential vegetation index to Anopheles density and malaria incidence rate. Biomed Environ Sci 2006, 19:130-132.

30. Bi P, Tong S, Donald K, Parton KA, Ni J: Climatic variables and transmission of malaria: a 12-year data analysis in Shuchen County, China. Public Health Rep 2003, 118:65-71

31. Hu H, Singhasivanon $P$, Salazar NP, Thimasarn K, Li X, Wu Y, Yang H, Zhu D, Supavej S, Looarecsuwan S: Factors influencing malaria endemicity in Yunnan Province, PR China (analysis of spatial pattern by GIS). Southeast Asian J Trop Med Public Health 1998, 29:191-200.

32. Liu TY, Shi M, Liu L, Zhang Y, Lou PA, Yuan FM, Shan Y: Analysis of the correlation between malaria and meteorological factors. Chin J Gen Pract 2011, 9:604-608.

33. Wu SM: Influence of meteorological factors on the incidence of malaria. Chinese and Foreign Medical Research 2011, 9:139-140.

34. Huang GQ, Zhang HX, Chen GY, Yuan FY, Pei SJ, Hu LQ, Chen JS, Lin W: Evaluation of malaria epidemic situation in Tongbai and Dabieshan Mountain Area in Hubei. Journal of Tropical Medicine 2009, 9:11.

35. Wang LP, Fang LQ, Xu X, Wang JJ, Ma JQ, Cao WC, Jin SG: Study on the determinants regarding malaria epidemics in Anhui province during 2004-2006. Chin J Epidemiol 2009, 30:38-41.

36. Wen L, Shi RH, Fang LQ, Xu DZ, Li CY, Wang Y, Yuan ZQ, Zhang H: Spatial epidemiological study on malaria epidemics in Hainan Province. Chin J Epidemio/ 2008, 29:581-585.

37. Su YQ, Zhang ZY, Xu DZ, Xi YZ, Wang SQ, Li CX: Factors analysis on the relationship of climatic variables, $\mathrm{NDVI}$, and malaria transmission in Hainan. J Prev Med Chin PLA 2006, 24:276-278.

38. Yu GW, Tang LH: Study on a grey model for evaluation of anopheles minimus density. Chin J Parasitol Parasit Dis 2005, 23:4.

39. Wen L, Xu DZ, Wang SQ, Li CX, Zhang ZY, Su YQ: Analysis on the relationship between malaria epidemics and NOAA-AVHRR NDVI in Hainan province. Zhonghua Liu Xing Bing Xue Za Zhi 2005, 26:263-267.

40. Huang SJ, Wang FC, Huang J, Feng L: Apllication of path analysis in studying climatic factors in malaria transmission. Journal of Qiannan Medical College for Nationalities 2004, 17:232-233.

41. Gao CX, Xiong HY, Yi D, Chai GJ, Yang XW, Liu L: Study on meteorological factors2based neural network model of malaria. Chin J Epidemiol 2003, 24:9.
42. Wen L, Xu DZ, Wang SQ, Li CX, Zhang ZY, Su YQ: Epidemic of malaria in Hainan Province and modeling malaria incidence with meteorological parameters. Chin J D is Control Prev 2003, 7:520-524.

43. Yang GJ, Zhou XN, Malone JB, Mccarroll JC, Wang TP, Liu JX, Gao Q, Zhang $X P$, Hong $Q B$, Sun LP: GIS prediction model of malaria transmission in Jiangsu province. Chin J Prev Med 2002, 36:2.

44. Huang HS: Analysis of seasonal pattern of malaria distribution in Gaoan City by circular distribution method. Modern Preventive Medicine 2001, 28:3.

45. Kan SP, Shen YZ, Zou Z, Xu FN: Studies on malaria epidemic characteristics and countermeasures in Anhui Province. Chinese Journal of Parasitic Disease Control 1999, 12:2.

46. Yu YS, Deng ZM, Huang SJ, Chu ZQ: Path analysis of climatic impacts on malaria. Chi J Health Statistics 1995, 12:3.

47. Wu F, Liu Q, Lu L, Wang J, Song X, Ren D: Distribution of Aedes albopictus (Diptera: Culicidae) in northwestern China. Vector Borne Zoonotic Dis 2011, 11:1181-1186.

48. Lai LW: Influence of environmental conditions on asynchronous outbreaks of dengue disease and increasing vector population in Kaohsiung, Taiwan. Int J Environ Health Res 2011, 21:133-146.

49. Chen SC, Liao CM, Chio CP, Chou HH, You SH, Cheng YH: Lagged temperature effect with mosquito transmission potential explains dengue variability in southern Taiwan: insights from a statistical analysis. Sci Total Enviroon 2010, 408:4069-4075.

50. Shang CS, Fang CT, Liu CM, Wen TH, Tsai KH, King CC: The role of imported cases and favorable meteorological conditions in the onset of dengue epidemics. PLoS Negl Trop Dis 2010, 4:e775.

51. Lu L, Lin H, Tian L, Yang W, Sun J, Liu Q: Time series analysis of dengue fever and weather in Guangzhou, China. BMC Publ Health 2009, 9:395.

52. Hsieh $\mathrm{YH}$, Chen $\mathrm{CW}$ : Turning points, reproduction number, and impact of climatological events for multi-wave dengue outbreaks. Trop Med Int Health 2009, 14:628-638.

53. Yang T, Lu L, Fu G, Zhong S, Ding G, Xu R, Zhu G, Shi N, Fan F, Liu Q: Epidemiology and vector efficiency during a dengue fever outbreak in Cixi, Zhejiang Province, China. J Vector Ecol 2009, 34:148-154.

54. Wu PC, Lay JG, Guo HR, Lin CY, Lung SC, Su HJ: Higher temperature and urbanization affect the spatial patterns of dengue fever transmission in subtropical Taiwan. Sci Total Environ 2009, 407:2224-2233.

55. Wu PC, Guo HR, Lung SC, Lin CY, Su HJ: Weather as an effective predictor for occurrence of dengue fever in Taiwan. Acta Trop 2007, 103:50-57.

56. Lu L, Lin HL, Liu QY: Risk map for dengue fever outbreaks based on meteorological factors. Adv. Clim. Change Res. 2010, 6(4):254-258.

57. Yu SX, Li ZQ, Teng WP, Cai J: Impact on the potential epidemic of dengue fever under warming winter in Hainan province. Chin J Epidemiol 2005 26:25-28.

58. Chen FY, Wu F, Lv ZP, He YM, Chen GX: Prediction research on dengue fever and vectors in South port of China. China J Frontier Health Quarantine 2003, 26:5-9.

59. Yi BT, Zhang ZY, Xu DZ, Xi YZ, Fu JG, Luo J, Yuan MH, Liu SQ, Kuang K: Influence of climate factors on vector aedes density of dengue. Chin $J$ Public Health 2003, 19:129-131.

60. Chen WJ, Li CX, Lin MH, Wu KS, KL W: Study on the suitable duration for dengue fever (DF) transmission in a whole year and potential impact on DF by global warming in Hainan Province. China Tropical Medicine 2002, 2:31-34

61. Zheng NX, Wang ZH, Zhang XY, Zheng G, Chen HH, Li WZ, Chen HG, Chen $M H$, Lin YQ, Zhao ST: Study on seasonal trend, propagating condition and the the influencing factors of Aedes albopictus in Fuzhou, China. Strait J Prev Med 2001, 7:6-9.

62. Lin H, Yang L, Liu Q, Wang T, Hossain SR, Ho SC, Tian L: Time series analysis of Japanese encephalitis and weather in Linyi City, China. Int J Public Health 2011, 57(2):289-296.

63. Bi $P$, Zhang $Y$, Parton KA: Weather variables and Japanese encephalitis in the metropolitan area of Jinan city, China. J Infect 2007, 55:551-556.

64. Hsu SM, Yen AM, Chen TH: The impact of climate on Japanese encephalitis. Epidemiol Infect 2008, 136:980-987.

65. Bi $\mathrm{P}$, Tong $\mathrm{S}$, Donald $\mathrm{K}$, Parton KA, Ni J: Climate variability and transmission of Japanese encephalitis in eastern China. Vector Borne Zoonotic Dis 2003, 3:111-115.

66. Huo AM, Zhao DS, Fang LQ, Cao WC: Association between infectious diseases with natural factors and meteorological factors in North China. Journal of Pathogen Biology 2011, 6:5-7. 
67. Xu L, Yang ZZ, Ran L, Fan QJ: Multiple stepwise regression analysis of relationship between disease occurred and meteorological factors. C J G MCM 2009, 24:993-995.

68. Gao L, Li X, Lu CG, Li XY, Yang JZ, Long FX, Jiang QY: Epidemic analysis of Japanese encephalitis in Guizhou Province. Modern Preventive Medicine 2009, 36:4608-4609.

69. Liu ZY, Du AG: Grey correlation analysis of meteorological factors and Japanese encephalitis incidences. Journal of Mathematical Medicine 2008, 22:64-66.

70. Qu B, Guo HQ, Guan P, Zhou BS, Huang DS: Study on the impact of meteorological factors on Japanese encephalitis incidence. Chin J Epidemiol 2006, 27:179.

71. Zhang YZ, Potjaman S, Zhang HL, Dong XQ, Wang SD, Huang QY, Chen BY, Chen HY: Situational analysis of Japanese Encephalitis in Dali Prefecture, Yunnan Province, China from 1992 to 2001. Endemic Diseases Bull etin 2004, 19:31-35.

72. Liu YJ, Deng Z, Zhao R, Hou WM, Li LH: Correlation analysis of Japanese encephalitis incidences and meteorological factors in Chaoyang City, Liaoning Province from 1983-2002. Journal of Tropical Disease and Parasitology 2003, 1:188.

73. Shen B, Ding D, Li YT, et al: Study on ecological factors for secular changes in incidence of Japanese Encephalitis in Shanghai. CHINA PUBLIC HEALTH 2002, 18(3):308-309.

74. Zhang YP, Li LC, Wu ZY, Guo WS, Wang WZ, Xia ZG, Zhao ZM, Que MJ: Study on geographical epidemiology of Japanese encephalitis in Henan Province. Henan Medical Research 1997, 6:69-73.

75. Feng $X G$, Zhang $H L$, Hong ZD, Li W: Epidemic investigation of Japanese encephalitis outbreaks in Eyuan County. Chinese Journal of Vector Biology and Control 1996, 7(5):365-366.

76. Zhou SS, Wang Y, Li Y: Malaria situation in the People's Republic of China in 2010. Chin J Parasitol Parasit Dis Dec. 2011, 29:6.

77. Gubler DJ, Reiter P, Ebi K, Yap W, Nasci R, Patz J: Climate variability and change in the United States: potential impacts on vector- and rodentborne diseases. Environ Health Perspect 2001, 109(Suppl. 2):223-233.

78. Qiu FX, Gubler DJ, Liu JC, Chen QQ: Dengue in China: a clinical review. Bull World Health Organ 1993, 71:349-359.

79. Jiang Y, Yan ZJ: Mechanism of dengue fever in the subtropical region of China. Chin J Vector Biol Control 2008, 19:80-81.

80. Xie H, Zhou HN, Yang YM: Advances in the research on the primary dengue vector Aedes aegypti in China. Chin J Vector Biol \& Control 2011, 22:2.

81. Sutherst RW: Global change and human vulnerability to vector-borne diseases. Clin Microbiol Rev 2004, 17:136-173.

82. Hales S, de Wet N, Maindonald J, Woodward A: Potential effect of population and climate changes on global distribution of dengue fever: an empirical model. Lancet 2002, 360:830-834.

83. Nagao Y, Thavara U, Chitnumsup P, Tawatsin A, Chansang C, CampbellLendrum D: Climatic and social risk factors for Aedesinfestation in rural Thailand. TropMed Int Health 2003, 8(7):650-659.

84. Watts DM, Burke DS, Harrison BA, Whitmire RE, Nisalak A: Effect of temperature on the vector efficiency of Aedes aegypti for dengue 2 virus. AmJTrop Med Hyg 1987, 36(1):143-152.

85. Kay BH, Fanning ID, Mottram P: Rearing temperature influences flavivirus vector competence of mosquitoes. Med Vet Entomol 1989, 3(4):415-422.

86. Turell MJ: Effect of environmental temperature on the vector competence of Aedes fowleri for Rift Valley fever virus. Res Virol 1989, 140(2):147-154.

87. Li HR: Epidemic encephalitis B. In Vector-Borne Infectious Diseases. Edited by Xu ZY. Ningxia, China: Ningxia People's Publishing House; 1990:85-95.

88. Wang HY, Li YX, Liang XF, Liang GD: Japanese encephalitis in Mainland China. Jpn J Infect Dis 2009, 62(5):331-336.

89. Wu YC: The epidemiology of Japanese encephalitis on Taiwan during 1966-1997. AmJTrop Med Hyg 1999, 61:78-84.

90. Li YX, Li MH, Fu SH, Chen WX, Liu QY, Zhang HL, Da W, Hu SL, Mu SD, Bai J, Yin ZD, Jiang HY, Guo YH, Ji DZ, Xu HM, Li G, Mu GG, Luo HM, Wang JL, Wang J, Ye XM, Jin ZM, Zhang W, Ning GJ, Wang HY, Li GC, Yong J, Liang XF, Liang GD: Japanese encephalitis, Tibet, China. Emerg Infect Dis 2011, 17:934-936

91. Van Den Hurk AF, Montgomery BL, Northill JA, Smith IL, Zborowski P, Ritchie SA, Mackenzie JS, Smith GA: Short report: the first isolation of
Japanese encephalitis virus from mosquitoes collected from mainland Australia. AmJTrop Med Hyg 2006, 75(1):21-25.

92. Erlanger TE, Weiss S, Keiser J, Utzinger J, Wiedenmayer K: Past, present, and future of Japanese encephalitis. Emerg Infect Dis 2009, 15(1):1-7.

93. Jeffrey NH, Scott AR, Debra AP, et al: An outbreak of Japanese encephalitis in the Torres Strait, Australia. Med J Aust 1998, 165:256-260.

94. Anyamba A, Chretien JP, Small J, Tucker CJ, Linthicum KJ: Developing global climate anomalies suggest potential disease risks for 2006-2007. Int J Health Geogr 2006, 5:60.

95. Houghton JT, Ding Y, Griggs DJ, Noguer M, Linden P, Dai X, Maskell K, Johnson CA: Climate change 2001: the scientific basis. In proceeding of working group I to the third assessment report of the intergovernmental panel on climate change (IPCC). Cambridge: Cambridge University Press; 2001.

96. Tong SL, Hu WB: Climate variation and incidence of Ross River virus in Cairns, Australia: a time-series analysis. Environ Health Perspect 2001, 109(12):1271-1273.

97. Khan S, Narain K, Handigue R, Dutta P, Mahanta J, Satyanarayana K: Role of some environmental factors in modulating seasonal abundance of potential Japanese encephalitis vectors in Assam, India. Southeast Asian J Trop Med Public Health 1996, 27:382-391.

98. Sakai T, Takahashi K, Hisasue S, Horimoto M, Takizawa T: Meteorological factors involved in Japanese encephalitis virus infection in cattle. Nippon Juigaku Zasshi 1990, 52:121-127.

99. Mogi M: Relationship between number of human Japanese encephalitis cases and summer meteorological conditions in Nagasaki, Japan. AmJTrop Med Hyg 1983, 32:170-174.

100. Weaver HJ, Blashki GA, Capon AG, McMichael AJ: Climate change and Australia's healthcare system - risks, research and responses. Aust Health Rev 2010, 34:441-444.

101. Füssel HM: Assessing adaptation to the health risks of climate change: what guidance can existing frameworks provide? Int J Environ Health Res 2008, 18:37-63.

102. Campbell-Lendrum D, Corvalán C: Climate change and developingcountry cities: implications for environmental health and equity. J Urban Health 2007, 84(Suppl 3):i109-i117.

103. Frumkin H, Hess J, Luber G, Mafilayt J, McGeehin M: Climate change: the public health response. Amer. J. Public Health 2008, 98:435-445.

104. McMichael AJ: Global environmental change as "risk factor": can epidemiology cope? Am J Public Health 2001, 91:1172-1174.

105. Haines A: Climate change and health: strengthening the evidence base for policy. Am J Prev Med 2008, 35:411-413.

106. Kovats RS, Campbell-Lendrum D, McMichael AJ, Woodward A, Cox J: Early effects of climate change: do they include changes in vector borne diseases? Philos T Roy Soc B 2001, 356:1057-1068.

107. Zhou SS, Tang LH, Sheng HF: Malaria situation in the People's Republic of China in 2003. Chin J Parasitol and Prasit Dis 2005, 23:385-387.

108. Haines A, McMichael AJ: Climate change and health: implications for research, monitoring, and policy. BMJ 1997, 315:870-874.

109. Anker M: Corval C(Eds): Using climate to predict infectious disease outbreaks: A review. Geneva: World Health Organization; 2004

110. Shu PY, Chien LJ, Chang SF, Su CL, Kuo YC: Fever screening at airports and imported dengue. Emerg Infect Dis 2005, 11:460-462.

111. Patz J, Campbell-Lendrum D, Gibbs H, Woodruff R: Health impact assessment of global climate change: expanding on comparative risk assessment approaches for policy making. Annu Rev Public Health 2008, 29:27-39.

112. Rosenthal JK, Sclar ED, Kinney PL, Knowlton K, Crauderueff R, Brandt-Rauf PW: Links between the built environment, climate and population health: interdisciplinary environmental change research in New York City. Ann Acad Med Singapore 2007, 36:834-846.

113. Zhang S, Xing HJ, Zhu CC: Present status of malaria control resources in China. Journal of Pathogen Biology 2010, 5(8):627-629.

114. Maibach EW, Chadwick A, McBride D, Chunk M, Ebi K, Balbus J: Climate change and local public health in the United stateds: preparedness, programs and perceptions of local public health department directors. PLoS One 2008, 3(7):1-8.

115. Watson RT: Turning science into policy: challenges and experiences from the science-policy interface. Philos Trans R Soc Lond B Biol Sci 2005, 360:471-477.

116. Millennium Ecosystem Assessment: Ecosystems and human well being: a framework for assessment. Washington, DC: Island Press; 2003. 
117. Ostry A, Ogborn M, Bassil KL, Takaro TK, Allen DM: Climate change and health in British Columbia: projected impacts and a proposed agenda for adaptation research and policy. Int J Environ Res Public Health 2010, 7:1018-1035.

118. Semenza JC, Ploubidis GB, George LA: Climate change and climate variability: personal motivation for adaptation and mitigation. Environ Health 2011, 10:46.

doi:10.1186/1744-8603-9-10

Cite this article as: Bai et al.: Climate change and mosquito-borne

diseases in China: a review. Globalization and Health 2013 9:10.

\section{Submit your next manuscript to BioMed Central and take full advantage of:}

- Convenient online submission

- Thorough peer review

- No space constraints or color figure charges

- Immediate publication on acceptance

- Inclusion in PubMed, CAS, Scopus and Google Scholar

- Research which is freely available for redistribution 